\title{
The effects of dust on the photometric parameters of decomposed disks and bulges *
}

\author{
Bogdan A. Pastrav ${ }^{1}$, Cristina C. Popescu ${ }^{1, \star \star}$, Richard J. Tuffs ${ }^{2}$, and Anne E. Sansom ${ }^{1}$ \\ 1 Jeremiah Horrocks Institute, University of Central Lancashire, PR1 2HE, Preston, UK \\ e-mail: [bapastrav; cpopescu; aesansom]@uclan.ac.uk \\ 2 Max Planck Institut für Kernphysik, Saupfercheckweg 1, 69117 Heidelberg, Germany \\ e-mail: Richard.Tuffs@mpi-hd.mpg.de
}

Received 14 June 2013 / Accepted 31 July 2013

\begin{abstract}
We present results of a study to quantify the effects of dust on the derived photometric parameters of disk and bulges obtained from bulge-disk decomposition: scale-length, effective radius, Sérsic index, disk axis-ratio, and bulge-to-disk ratio. The dust induced changes in these parameters were obtained by fitting simulated images of composite systems (containing a disk and a bulge) produced using radiative transfer calculations. The simulations were fitted with the GALFIT 3.0.2 data analysis algorithm. Fits were done with both a combination of an exponential plus a variable-index Sérsic function as well as with a combination of two variable-index Sérsic functions. We find that dust is biasing the derived exponential scale-length of decomposed disks towards smaller values than would be otherwise derived if the galaxy were to have no bulge. Similarly, the derived bulge-to-disk ratio is biased towards smaller values. However, the derived axis-ratio of the disk is not changed in the decomposition process. The derived effective radius of decomposed disks of systems having exponential bulges is found to be less affected by dust when fits are done with two variable-index Sérsic functions. For the same type of fits dust is found to bias the value of the derived effective radius of decomposed disks towards lower values for systems having de Vaucouleurs bulges.
\end{abstract}

Key words. galaxies: spiral - galaxies: photometry - galaxies: bulges - galaxies: structure - dust, extinction - radiative transfer

\section{Introduction}

Spiral galaxies are complex systems containing two primary, physically distinct morphological components: a disk and a bulge. The bulge is a predominantly pressure-supported spheroidal component containing old stellar populations. Being pressure supported, there can be no substantial cold interstellar medium associated with the spheroid. Consequently, it is believed that there is no dust associated with this component. Conversely, the disk is a flat, rotationally-supported component containing young, intermediate-age and old stellar populations, with star-formation activity mainly occurring in a system of spiral arms. Unlike the bulge, the disk is associated with a cold interstellar medium, and contains large amounts of dust. The dust in the disk has the effect of attenuating the stellar light from both the disk and the bulge (e.g. Tuffs et al. 2004; Driver et al. 2007).

Although the bimodal structure of spiral galaxies has long been known, the separate evolutionary history of these two morphological components, in terms of when and how they acquired their present-day stellar populations, is still poorly understood. One reason for this is that, observationally, it is difficult to trace the independent evolutionary history of disks and bulges, as this requires bulge-disk decompositions to be performed on higher resolution images of galaxies in large statistical samples.

\footnotetext{
* Tables with corrections derived in this paper are only available at the CDS via anonymous ftp to

cdsarc.u-strasbg.fr (130.79.128.5) or via

http://cdsarc.u-strasbg.fr/viz-bin/qcat?]/A+A/557/A137

$\star \star$ Visiting Scientist at the Max Planck Institut für Kernphysik, Saupfercheckweg 1, 69117 Heidelberg, Germany.
}

Such analyses have been lacking until recently, so that studies of decomposed bulges and disks have been mainly restricted to small samples of highly resolved local universe galaxies (e.g. Möllenhoff et al. 1999; Möllenhoff \& Heidt 2001; Möllenhoff 2004; Fisher \& Drory 2008; Fabricius et al. 2012). The situation is now rapidly changing, with the advent of deep wide-field spectroscopic and photometric surveys of galaxies (e.g. SDSS, York et al. 2000; GAMA, Driver et al. 2011), which are providing us with large samples of galaxies for which major morphological components can be resolved out to $z=0.1$. This trend will continue into the future with the advent of new ground based surveys such as The VST Atlas, The Kilo Degree Survey (KiDS; de Jong et al. 2012), the Dark Energy Survey (DES; The DES collaboration 2005), which will provide wide-field imaging surveys with sub-arcsec resolution, and will culminate in the wide-field diffraction-limited space-borne surveys made with EUCLID (Laureijs et al. 2010). In parallel, automatic routines such as GALFIT (Peng et al. 2002, 2010), GIM2D (Simard et al. 2002), BUDDA (Gadotti 2008) or MegaMorph (Häußler et al. 2013; Vika et al. 2013) have been developed to address the need to fit large numbers of images of galaxies with 1D analytic functions for the characterisation of the surface brightness distributions of their stellar components. In particular these routines allow bulge-disk decomposition to be performed routinely, as already done by Allen et al. (2006); Benson et al. (2007); Cameron et al. (2009); Gadotti (2009); Simard et al. (2011); Lackner \& Gunn (2012); Bruce et al. (2012), and Bernardi et al. (2012).

One potential problem with the results coming from bulgedisk decomposition is that the available routines that are commonly used to perform surface-brightness photometry cannot 
take into account the effects of dust. It is already known that spiral galaxies contain large amounts of dust (Stickel et al. 2000, 2004; Tuffs et al. 2002; Popescu et al. 2002; Vlahakis et al. 2005; Driver et al. 2007; Dariush et al. 2011; Rowlands et al. 2012; Bourne et al. 2012; Dale et al. 2012; Grootes et al. 2013a), and that this dust changes the appearance of disks and bulges from what would be predicted based solely on their intrinsic stellar distributions (e.g. Tuffs et al. 2004; Möllenhoff et al. 2006; Gadotti et al. 2010; Pastrav et al. 2013). Since the routines available to fit galaxy images use simple analytic functions, most commonly Sérsic functions, the fits to real images will be imperfect, resulting in an over- or under-estimation of the parameters corresponding to the projected stellar distributions.

In Pastrav et al. (2013) we gave a detailed account of the different applications that require accurate knowledge of the intrinsic photometric parameters of galaxies (i.e. corrected for the effect of dust). Here we add relevant applications that have been emphasised by recent work. Thus, Casaponsa et al. (2013) showed that cosmic size magnification can be used to complement cosmic shear in weak gravitational lensing surveys, with a view to obtaining high-precision estimates of cosmological parameters. Thus, not only modification of the galaxy shape (i.e. axis ratios) - a measure of the shear - can be used in studies of weak lensing, but, for space-based data with $0.1-0.2 \operatorname{arcsec}$ resolution, the size distribution of galaxies may be an important tool for determining cosmic size magnification. In view of this, it is extremely important to estimate the effects of dust on the scale-lengths of galaxies. Sérsic indices of bulges are also important, as they provide a link to their supermassive black hole. Following on the work of Graham et al. (2001) and Graham \& Driver (2007a), recent work by Savorgnan et al. (2013) found a clear supermassive black-hole mass - Sérsic index relation. Thus, if accurate Sérsic indices can be derived (corrected for the effect of dust and for projection effects), then these can be used to predict black hole masses in large samples of galaxies to derive the local black hole mass function (e.g. Graham et al. 2007) and space density (Graham \& Driver 2007b). Dust corrections are also important on scaling relation in galaxies in general. Thus, Grootes et al. (2013b) have recently shown that, by applying dust corrections from Popescu et al. (2011) and from the present paper, the scatter in the scaling relation specific star-formation rate versus stellar mass can be reduced from 0.58 dex to 0.37 dex.

In this paper we quantify the effect of dust on the photometric parameters of decomposed bulges and disks of spiral galaxies. As discussed in Pastrav et al. (2013), this effect can be separated from the effect of dust on disks and bulges taken individually, as seen through a common distribution of dust. Overall, when performing surface-brightness photometry there are three corrections that should be taken into account: projection effects on disks and bulges viewed individually; the effects of dust on disks and bulges viewed individually; and the projection and dust effects on the disks and bulges viewed in combination.

Projection effects arise even in the absence of dust, causing the fitted functions to imperfectly recover the structure of real disks and bulges due to the fact that these functions describe infinitely thin templates, in contrast to real disks and bulges, which have a thickness. Thus, the additional vertical distribution of stars superimposed on the radial distribution produces isophotal shapes which differ from those predicted by an infinitely thin template. The correction for projection effects on disks and bulges seen in isolation is needed to transform the derived photometric parameters obtained from fitting dustless images of disks and bulges to those characterising the volume stellar emissivity. Corrections for this effect have been given in Pastrav et al. (2013).

The second type of correction that needs to be taken into account when performing surface-brightness photometry is due to the effects of dust on disks and bulges when viewed individually. Such effects arise because dust distorts the appearance of disks and bulges. This leads to a discrepancy between the derived photometric parameters of dust-attenuated disks and bulges and the parameters that would be derived for disks and bulges if they could be seen at the same inclination, but in the absence of dust. Corrections for this discrepancy have been given for pure disks by Möllenhoff et al. (2006) (albeit without separately considering the projection effects), and for both disks and bulges by Pastrav et al. (2013).

The third type of correction relates to the joint projection and dust effect on disks and bulges viewed in combination, attention to which was first drawn by Gadotti et al. (2010). This effect causes the decomposed attenuated disk and decomposed attenuated bulge fitted with infinitely thin and dustless templates to differ from the appearance of the real dust-attenuated disk and bulge. In other words the decomposed dust-attenuated disk in the presence of a bulge may be imperfectly subtracted and therefore differ from the dust-attenuated disk that would be fitted if the galaxy were to have no bulge. Conversely, the decomposed dust-attenuated bulge in the presence of a disk may also be imperfectly subtracted and differ from how it would appear in reality if it could be seen in the absence of the stellar disk. Of course these artifacts are specific to routines that perform bulge-disk decomposition using simple analytical infinitely thin dustless templates. However, this is the common practice, as it is the only feasible approach at present.

As mentioned before, in Pastrav et al. (2013) we quantified the projection effects and the effects of dust on disks and bulges viewed in isolation. Here we consider the joint projection and dust effects on disks and bulges viewed in combination, thus completing the tool kit needed to fully correct the derived photometric parameters. As proposed in Pastrav et al. (2013), these three effects can be multiplied (or added) together (depending on the photometric parameter considered) using a chain correction approach, to derive total corrections needed to convert the photometric parameters obtained from bulge-disk decomposition of spiral galaxies into those of the volume stellar emissivity. Here and in Pastrav et al. (2013) we used simulations based on a radiation transfer model that can simultaneously account for both dust-attenuation in the ultraviolet (UV)/optical range and dust emission in the mid-infrared (MIR)/far-infrared (FIR)/submillimeter (sub-mm) range. Most of the simulations come from the library of Popescu et al. (2011), while additional simulations have been created for analysis in Pastrav et al. (2013). In this paper we provide a comprehensive data set of corrections for decomposed disks and bulges that cover the whole parameter range in dust opacity, inclination and wavelength. The corrections are also provided for two different values of bulge-to-disk ratios. All the corrections are made publically available at the CDS database.

This paper is organised as follows. In Sect. 2, we briefly describe the stellar emissivity and dust distributions used in the simulations. The method and general approach used to fit the simulated images of the galaxies and derive the apparent photometric parameters of the decomposed disks and bulges is explained in Sect. 3, while the technical details of the whole fitting process are presented in Sect. 4. In Sect. 5 we quantify the projection effects on the bulge-disk decomposition process, while the dust effects on disks and bulges seen in combination are 
given in Sect. 6. Single Sérsic fits to the same simulated images are presented in Sect. 7. In Sect. 8 we present an application of our predictions for the inclination dependence of dust effects, while in Sect. 9 we summarise our results.

\section{Simulated images}

Our simulated images are those used to generate the library of UV/optical dust attenuations first presented in Tuffs et al. (2004) and then in updated form in Popescu et al. (2011). This library is self-consistently calculated with the corresponding library of dust- and polycyclic aromatic hydrocarbon (PAH)-emission spectral energy distributions (SEDs) given in Popescu et al. (2011). Additional simulations used in this work were presented in Pastrav et al. (2013). The calculations are described at length in Popescu et al. (2011). Here we only briefly mention their main characteristics. All simulations were made using a modified version of the ray-tracing radiative transfer code of Kylafis \& Bahcall (1987) and the dust model from Weingartner \& Draine (2001) and Draine \& Li (2007) incorporating a mixture of silicates, graphites and PAH molecules. The simulations were produced separately for old stellar disks, bulges and young stellar disks, all seen through a common distribution of dust (in the disks). The geometrical model of Popescu et al. (2011) consists of both a large scale distribution of diffuse dust and stars, as well as a clumpy component physically associated with the star-forming complexes. For the purpose of this study only the large scale distribution of diffuse dust is considered, as it is this that affects the large-scale distribution of UV/optical light determining the values of parameters typically used in fitting surfacebrightness distributions.

The intrinsic distributions of volume stellar emissivity are described by exponential functions in both the radial and vertical direction for the disks, and by deprojected Sérsic functions for the bulges. The corresponding dust distributions are described by double (radial and vertical) exponential functions for the two dust disks of the model. A schematic representation of the geometrical model can be found in Fig. 1 from Popescu et al. (2011). All the simulated images are sampled at $34.54 \mathrm{pc} / \mathrm{pixel}$. The images of the individual morphological components were analysed in Pastrav et al. (2013) to quantify both the projection effects and the effects of dust on the photometric parameters of each component.

To quantify the projection and dust effects on bulge-disk decompositions, the simulated images of the old stellar disk and bulges were summed to create simulated images of galaxies, for a set of values of disk inclination, wavelength, dust opacity, and bulge-to-disk ratios. The set of values span the whole parameter space of the model of Popescu et al. (2011). Thus simulations were produced for seven values of central face-on $B$-band optical depth $\tau_{B}^{f}$ (plus the dustless case), 21 values for the disk inclination $i$, and five wavelengths corresponding to the standard optical/near-infrared (NIR) bands $B, V, I, J, K$. We also consider two values of the bulge-to-disk ratio, $B / D=0.25,0.5$, where $B / D$ is the ratio of the luminosity of a single dust attenuated bulge and disk. In other words $B / D$ is the apparent bulge-todisk ratio (if both disk and bulges could be seen in isolation). As we will show in this paper the corrections for projection and dust effects on bulge-disk decomposition only show a mild dependence on the bulge-to-disk ratio, therefore there was no need to sample more finely the parameter $B / D$. For other values of the $B / D$ ratio corrections for bulge-disk decomposition can be obtained by interpolating between $B / D=(0,0.25,0.5)$ for disks and between $B / D=(0.25,0.5, \infty)$ for bulges. We note that when total corrections are derived to transform apparent parameters obtained from bulge-disk decomposition into intrinsic parameters of the stellar volume emissivity, interpolation can be performed between four values of the bulge-to-disk ratio, $B / D=(0,0.25,0.5, \infty)$, where corrections for $B / D=0$ and $B / D=\infty$ correspond to those of single disks and bulges, respectively.

We consider both exponential and de Vaucouleurs bulges. The values of the central face-on $B$-band dust optical depth cover a wide range, from almost dustless to extremely optically thick cases, $\tau_{B}^{f}=0.1,0.3,0.5,1.0,2.0,4.0,8.0$. Inclination was sampled according to $\triangle \cos (i)=0.05$, with $1-\cos (i) \in[0,1]$, resulting in 21 values.

\section{Method}

We follow the same procedure observers apply in bulge-disk decompositions of real images of galaxies and perform a multicomponent fit of the simulated images with two planar template shapes (commonly referred to as "infinitely thin disks"), one for each morphological component. The functions used to describe these shapes are the exponential function:

$\Sigma(r)=\Sigma_{0} \exp \left(-\frac{r}{r_{\mathrm{s}}}\right)$

and the variable-index Sérsic function

$\Sigma(r)=\Sigma_{0} \exp \left[-\kappa_{n}\left(\frac{r}{r_{\mathrm{e}}}\right)^{1 / n}\right]$

where $\Sigma_{0}$ is the central surface brightness, $r_{\mathrm{s}}$ represents the exponential scale-length of the template, $r_{\mathrm{e}}$ denotes the effective radius (enclosing half the total flux) of the template, $n$ is the Sérsic index, and $\kappa_{n}$ is a constant, coupled with $n$ (Ciotti \& Bertin 1999; Graham \& Driver 2005).

We consider the following types of fits: i) fits combining the exponential function (Eq. (1)) and the variable-index Sérsic function (Eq. (2)) for the disk and bulge component, respectively; and ii) fits combining two variable-index Sérsic functions for both the disk and the bulge.

As described in Pastrav et al. (2013), our approach is to separate projection effects on disks and bulges seen individually, the effects of dust on disks and bulges viewed individually and the joint projection and dust effects on disks and bulges viewed in combination (see Eqs. (4)-(11) in Pastrav et al. 2013). For each of these effects we present the results as corrections which can be used by observers separately or in combination. While the first two types of corrections were quantified and discussed in our previous work, in this paper we derive the third set of corrections, needed to quantify the influence of projection effects and dust on the decomposition process. These are presented as ratios (for extrinsic quantities; see Eqs. (3)-(5) below) or differences (for intrinsic quantities; Eqs. (6)-(7) below) between the fitted parameters obtained from bulge-disk decomposition in the presence of dust $R_{\mathrm{app}, \mathrm{d}}^{B / D}, R_{\mathrm{app}, \mathrm{d}}^{\mathrm{eff}, B / D}, R_{\mathrm{app}, \mathrm{b}}^{\mathrm{eff}, B / D}, n_{\mathrm{app}, \mathrm{d}}^{\text {sers, } B / D}, n_{\mathrm{app}, \mathrm{b}}^{\text {sers, } B D}$ (the measured parameters of the decomposed disk/bulge), and the fitted parameters of the same disk/bulge if these were to be observed as single components $R_{\text {app,d }}, R_{\mathrm{app}, \mathrm{d}}^{\text {eff }}, R_{\mathrm{app}, \mathrm{b}}^{\text {eff }}, n_{\mathrm{app}, \mathrm{d}}^{\mathrm{sers}}$, $n_{\text {app, b }}^{\text {sers }}$ (already measured in Pastrav et al. 2013), through the same distribution of dust. Thus, the correction for the exponential scale-length of the decomposed disk fitted with an exponential function, $\operatorname{corr}^{B / D}\left(R_{\mathrm{d}}\right)$, is

$\operatorname{corr}^{B / D}\left(R_{\mathrm{d}}\right)=\frac{R_{\mathrm{app}, \mathrm{d}}^{B / D}}{R_{\mathrm{app}, \mathrm{d}}}$, 
the corrections for the effective radii of decomposed disks and bulges fitted with variable-index Sérsic functions, $\operatorname{corr}^{B / D}\left(R_{\mathrm{d}}^{\text {eff }}\right)$ and $\operatorname{corr}^{B / D}\left(R_{\mathrm{b}}^{\text {eff }}\right)$, are

$\operatorname{corr}^{B / D}\left(R_{\mathrm{d}}^{\mathrm{eff}}\right)=\frac{R_{\mathrm{app}, \mathrm{d}}^{\mathrm{eff}, B / D}}{R_{\mathrm{app}, \mathrm{d}}^{\mathrm{eff}}}$

$\operatorname{corr}^{B / D}\left(R_{\mathrm{b}}^{\mathrm{eff}}\right)=\frac{R_{\mathrm{app}, \mathrm{b}}^{\mathrm{eff}, B / D}}{R_{\mathrm{app}, \mathrm{b}}^{\mathrm{eff}}}$,

with $d=$ disk and $b=$ bulge, and the corrections for the corresponding Sérsic index, $\operatorname{corr}^{B / D}\left(n_{\mathrm{d}}^{\text {sers }}\right)$ and $\operatorname{corr}^{B / D}\left(n_{\mathrm{b}}^{\text {sers }}\right)$, are

$\operatorname{corr}^{B / D}\left(n_{\mathrm{d}}^{\text {sers }}\right)=n_{\text {app, } \mathrm{d}}^{\text {sers } B / D}-n_{\text {app, } \mathrm{d}}^{\text {sers }}$

$\operatorname{corr}^{B / D}\left(n_{\mathrm{b}}^{\text {sers }}\right)=n_{\mathrm{app}, \mathrm{b}}^{\text {sers } B / D}-n_{\mathrm{app}, \mathrm{b}}^{\text {sers }}$.

We note here that the corrections measured by Eqs. (3)-(7) include both a component due to dust as well as a component due to projection effects. Unlike the corrections measured on single components, it is not possible to only measure a dust effect on the decomposition. However, we can measure pure projection effects on the decomposition process, by comparing similar quantities without dust.

Thus, the corresponding corrections due to pure projection effects are presented as ratios (see Eqs. (8)-(10) below) or differences (Eqs. (11), (12) below) between the fitted parameters obtained from bulge-disk decomposition in the absence of dust $R_{i, \mathrm{~d}}^{B / D}, R_{i, \mathrm{~d}}^{\text {eff, } B / D}, R_{i, \mathrm{~b}}^{\text {eff, } B / D}, n_{i, \mathrm{~d}}^{\text {sers, } B / D}, n_{i, \mathrm{~b}}^{\text {sers, } B / D}$ (the measured parameters of the decomposed disk/bulge), and the fitted parameters of the same disk/bulge if these were to be observed as single components $R_{i, \mathrm{~d}}, R_{i, \mathrm{~d}}^{\text {eff }}, R_{i, \mathrm{~b}}^{\mathrm{eff}}, n_{i, \mathrm{~d}}^{\text {sers }}, n_{i, \mathrm{~b}}^{\text {sers }}$ (already measured in Pastrav et al. 2013), again in the absence of dust. Thus, the correction for projection effects on the exponential scale-length of the decomposed disk fitted with an exponential function, corr $^{\operatorname{proj}, B / D}\left(R_{\mathrm{d}}\right)$, is

$\operatorname{corr}^{\mathrm{proj}, B / D}\left(R_{\mathrm{d}}\right)=\frac{R_{i, \mathrm{~d}}^{B / D}}{R_{i, \mathrm{~d}}}$,

the corrections for the effective radii of decomposed disks and bulges fitted with variable-index Sérsic functions, $\operatorname{corr}^{\operatorname{proj}, B / D}\left(R_{\mathrm{d}}^{\mathrm{eff}}\right)$ and $\operatorname{corr}^{\mathrm{proj}, B / D}\left(R_{\mathrm{b}}^{\mathrm{eff}}\right)$, are

$\operatorname{corr}^{\operatorname{proj}, B / D}\left(R_{\mathrm{d}}^{\mathrm{eff}}\right)=\frac{R_{i, \mathrm{~d}}^{\mathrm{eff}, B / D}}{R_{i, \mathrm{~d}}^{\mathrm{eff}}}$

$\operatorname{corr}^{\mathrm{proj}, B / D}\left(R_{\mathrm{b}}^{\mathrm{eff}}\right)=\frac{R_{i, \mathrm{~b}}^{\mathrm{eff}, B / D}}{R_{i, \mathrm{~b}}^{\mathrm{eff}}}$,

with $d=$ disk and $b=$ bulge, and the corrections for the corresponding Sérsic index, $\operatorname{corr}^{\text {proj, } B / D}\left(n_{\mathrm{d}}^{\text {sers }}\right)$ and $\operatorname{corr}^{\text {proj, } B / D}\left(n_{\mathrm{b}}^{\text {sers }}\right)$, are

$\operatorname{corr}^{\mathrm{proj}, B / D}\left(n_{\mathrm{d}}^{\mathrm{sers}}\right)=n_{i, \mathrm{~d}}^{\mathrm{sers}, B / D}-n_{i, \mathrm{~d}}^{\mathrm{sers}}$

$\operatorname{corr}^{\mathrm{proj}, B / D}\left(n_{\mathrm{b}}^{\text {sers }}\right)=n_{i, \mathrm{~b}}^{\text {sers, } B / D}-n_{i, \mathrm{~b}}^{\text {sers }}$.

The corrections for projection effects can be subtracted from the measurements that provide joint corrections, to isolate pure dust effects, $c o r r^{\text {dust, } B / D}$ on the decomposition process. We can then write:

$\operatorname{corr}^{\mathrm{dust}, B / D}=\operatorname{corr}^{B / D}-\operatorname{corr}^{\mathrm{proj}, B / D}$.
In addition to two-component fits to galaxies with two components, we also performed single Sérsic (sS) fits to the same simulated images (of galaxies with two components). This part of our study was motivated by the fact that real images of galaxies are still commonly being analysed by observers using global Sérsic fits to obtain their radial sizes. Since the prime motivation for this is the derivation of disk sizes, we only give corrections $\left(\operatorname{corr}^{\mathrm{SS}}\left(R_{\mathrm{gal}}\right)\right)$ as ratios between effective radii obtained from single Sérsic fits of dusty galaxies containing bulges, and the effective radii of corresponding dusty disks (derived from variable-index Sérsic fits to the pure disks with no bulges):

$\operatorname{corr}^{\mathrm{sS}}\left(R_{\mathrm{gal}}\right)=\frac{R_{\text {app, gal }}^{\mathrm{eff}}}{R_{\text {app }, \mathrm{d}}^{\mathrm{eff}}}$.

This isolates the effect of the bulge presence in constraining disk sizes from single Sérsic fits. The correction from Eq. (14) can be used in combination with the corrections for dust and projection effects on single disks (Eq. (4) from Pastrav et al. 2013) to relate the effective radius of a disk derived from single Sérsic fits to the intrinsic effective radius of the stellar emissivity in the disk through the chain corrections:

$\operatorname{corr}=\operatorname{corr}^{\mathrm{proj}} * \operatorname{corr}^{\mathrm{dust}} * \operatorname{corr}^{\mathrm{sS}}$.

All corrections are presented in terms of polynomial fits. The fits are of the form:

$\operatorname{corr}(x)=\sum_{k=0}^{N} a_{k} x^{k}$ for $0 \leq x \leq 0.95$,

where $x=1-\cos (i)$ and $\mathrm{N}$ has a maximum value of 5 . Although the polynomial fits extend to 0.95 , we note here that beyond $x=0.6$ the fits become progressively poorer, due to projection effects.

\section{Fitting procedure}

Following Pastrav et al. (2013) we used the GALFIT (version 3.0.2) data analysis algorithm (Peng et al. 2002, 2010) to fit our simulated images. GALFIT uses a non-linear least squares fitting based on the Levenberg-Marquardt algorithm, whereby the goodness of the fit is checked by computing the $\chi^{2}$ between the simulated image (in the case of observations, the real galaxy image) and the model image (created by GALFIT to fit the galaxy image). This is an iterative process, and the free parameters corresponding to each component are adjusted after each iteration in order to minimise the normalized (reduced) value of $\chi^{2}\left(\chi^{2} / N_{\text {DOF }}\right.$, with $N_{\text {DOF }}=$ number of pixels-number of free parameters, being the number of degrees of freedom).

Since our simulated images are noiseless, we use as input to GALFIT a "sigma" image (error/weight image) which is constant for all pixels, except for points outside the physical extent of our simulated galaxies. The latter points were set to a very high value, to act as a mask. This was necessary since our simulations are truncated in their volume stellar and dust emissivities while the fitting functions extend to infinity. We did not try to use the truncation functions from GALFIT, as these truncations are for the surface-brightness distributions, rather than for the volume stellar emissivity, as used when creating our simulated images. The model images have no background (by construction, unlike real images); accordingly the sky value was set to zero during the fitting procedure.

The free parameters of the two-component fits are: the $Y$ coordinate of the centre of the galaxy in pixels (while this is a free 

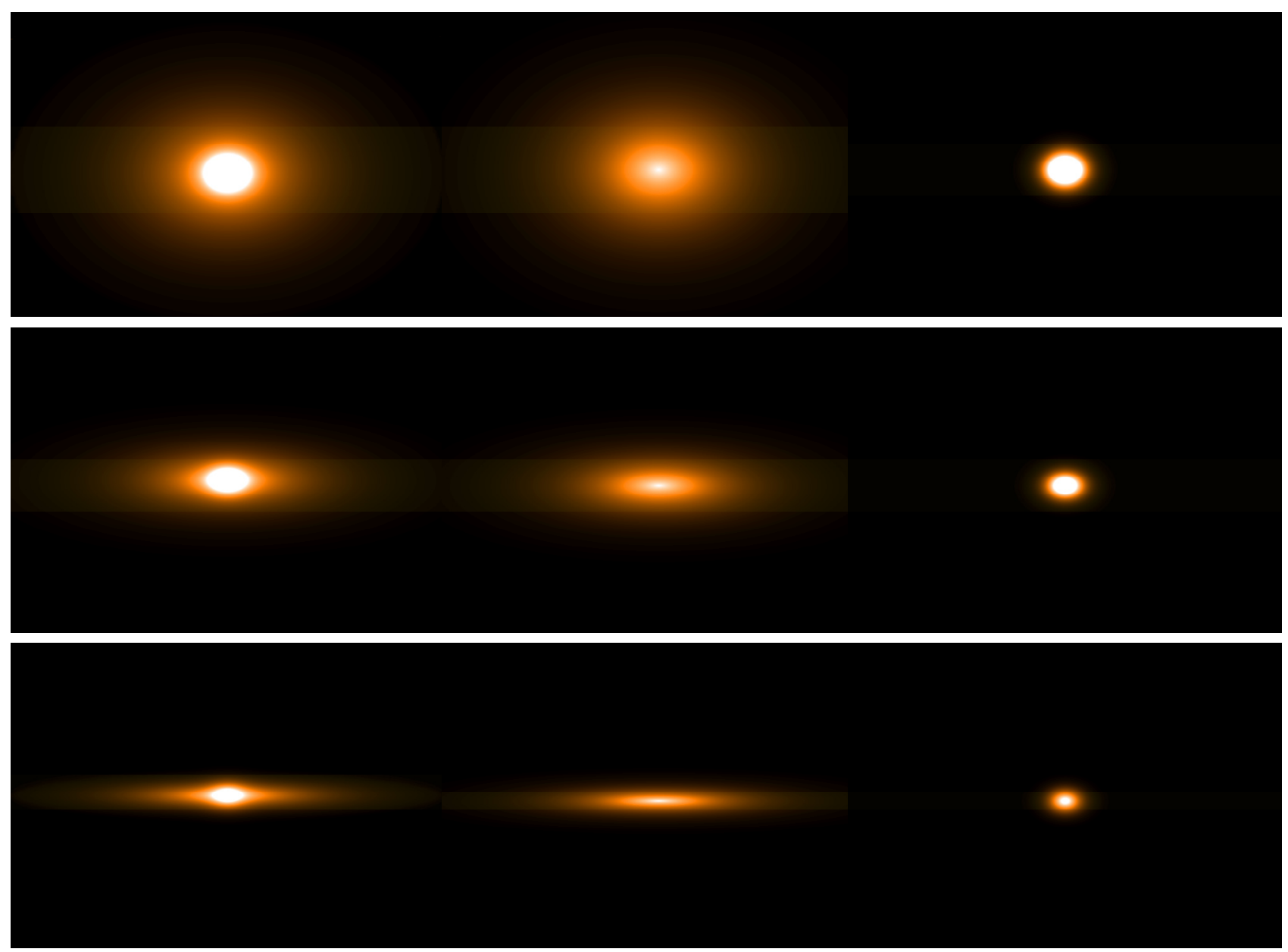

Fig. 1. Simulated images of dustless galaxies with exponential bulges and $B / D=0.25$ (left column) and corresponding decomposed disks and bulges (middle and right columns). The bulge-disk decomposition fit was made with an exponential plus a variable index Sérsic function, at inclinations $1-\cos (i)=0.3,0.7,0.9\left(i=46^{\circ}\right.$ (first row), $73^{\circ}$ (second row) and $84^{\circ}$ (third row)).

parameter, it is however constrained to be the same for both the disk and the bulge component), the integrated magnitudes of the disk and bulge components, the scale-length/effective radius (for exponential/Sérsic function), axis-ratios, and Sérsic index (for Sérsic function). The axis-ratio is defined as the ratio between the semi-minor and semi-major axis of the model fit (for each component). The position angle is the angle between the semimajor axis and the $Y$ axis (increasing counter clock-wise). For all our simulated images, the position angle was fixed to -90 (semi-major axis perpendicular on $Y$ axis).

\section{Projection effects on the bulge-disk decomposition}

As explained in Pastrav et al. (2013), even in the absence of dust, the derived photometric parameters of the images measured from fitting infinitely thin disk distributions would differ from the intrinsic parameters of the volume stellar emissivity due to the thickness (vertical stellar distribution) of real galaxies, which we called projection effects. These effects also act on the bulgedisk decomposition, causing the decomposed disks and bulges to differ in appearance from single disks and single bulges. In other words, projection effects are a further reason, apart from changes in morphology due to dust, through which the decomposed disk in the presence of a bulge may be imperfectly subtracted and therefore differ from the disk that would be fitted if the galaxy had no bulge. Conversely, the decomposed bulge in the presence of a disk may also be imperfectly subtracted and differ from how it would appear in reality if it could be seen in the absence of the stellar disk, due to projection effects.

\subsection{Galaxies with exponential bulges}

\subsubsection{Fits with exponential + variable-index Sérsic functions}

The first type of fit performed on the two-component simulated dustless galaxies involves fitting a superposition of an exponential plus a variable-index Sérsic function for the disk and bulge component, respectively. Examples of bulge-disk decompositions performed in this way are given in Fig. 1, for a bulgeto-disk ratio $B / D=0.25$. In Fig. 2 we also show results from these fits in the form of major- and minor-axis profiles (upper and middle rows) and relative residuals (bottom row).

To understand the trends due to projection effects on bulgedisk decomposition, one needs to compare them with the similar effects produced on fits of single components, as described in Pastrav et al. (2013). Thus, we showed in our previous paper that projection effects on single bulges manifest equally at all inclinations, and act to lower the measured Sérsic indices and to increase the measured effective radii with respect to the corresponding intrinsic parameters of the volume stellar emissivity (see Figs. 5 and 6 from Pastrav et al. 2013). When a disk is also 

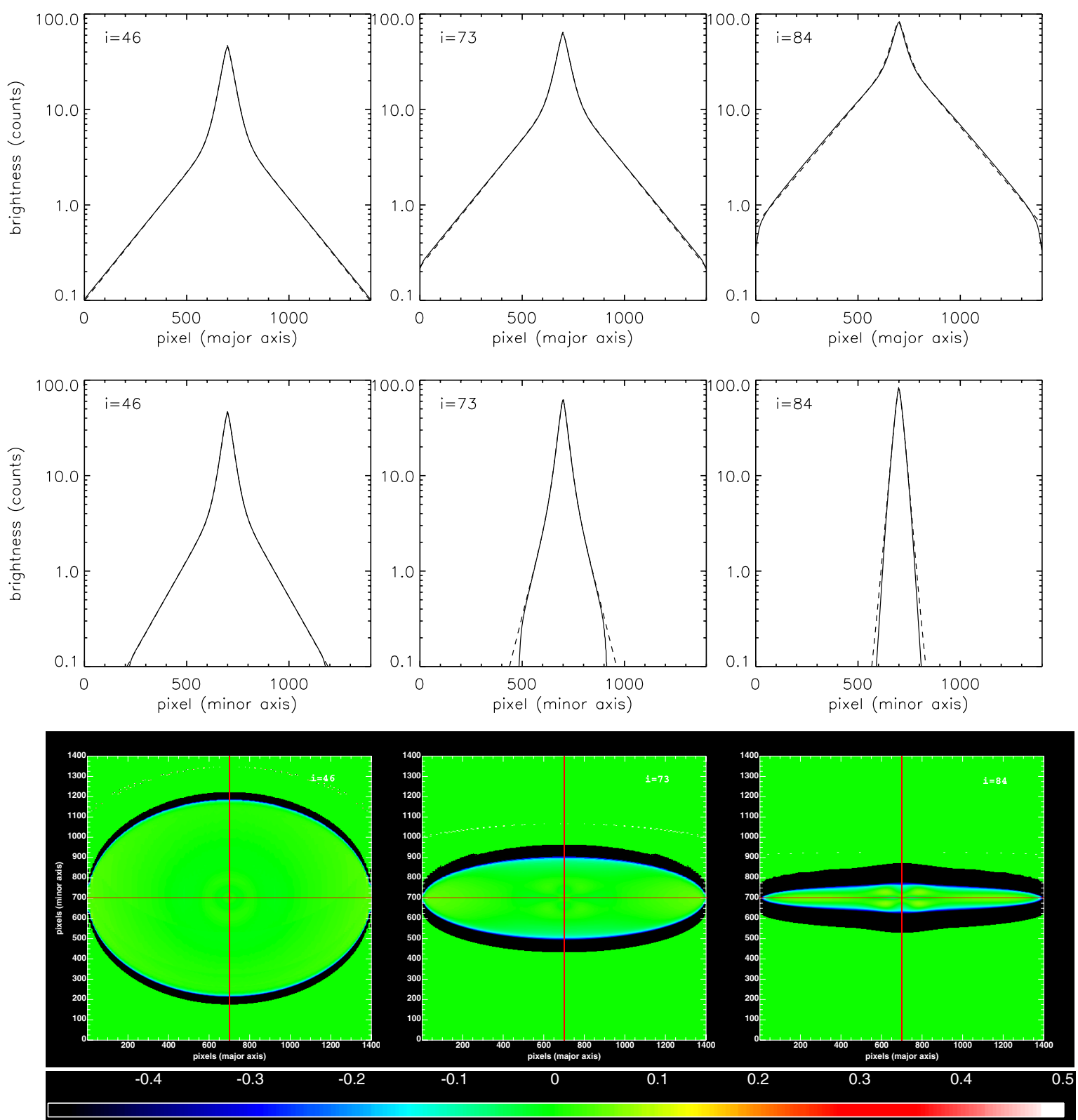

Fig. 2. Major- and minor-axis profiles of dustless galaxies (upper and middle rows) with $B / D=0.25$, in the $B$-band. Fits are made with an exponential function (for the disk component) and a variable-index Sérsic function (for the exponential bulge). Solid and dashed curves are for simulations and corresponding fits, respectively. The cuts were taken parallel and perpendicular to the major-axis of the simulated image, through the intensity peak, at inclinations $1-\cos (i)=0.3,0.7,0.9\left(i=46^{\circ}, 73^{\circ}, 84^{\circ}\right)$. Lower row: corresponding relative residuals $\left(\frac{\text { simulation-fit }}{\text { simulation }}\right)$, at the same inclination as the profiles. The red lines show radial and vertical cuts through the geometrical centre of the image.

present, we will see here that the small deviations from the exponential form of the bulge will cause some transfer of light from the bulge to the disk. This will cause the exponential fit to the disk to overpredict the amplitude of the light in the centre and to underpredict the scale-length, and conversely will cause the fit to the bulge to underpredict the amplitude of the light in the centre of the bulge and overpredict the effective radius. The effect on the integrated light of the changes in the central amplitude outweigh the effects of the changes in scale-length/effective radius, so that the projection effects lead to a measured bulge-to-disk ratio slightly smaller than the one corresponding to the bulge and the disk fitted individually.

One can see the trend of overestimating the light in the centre of decomposed disks when plotting the major- and minor-axis profiles of the fitted decomposed disks and corresponding simulated single disks (Fig. 3, for $B / D=0.25$ ). Even at low inclinations the fitted disks show an excess of light in the centre (at $i=46^{\circ}$ ). Because the scale-length of the fitted decomposed disk will be slightly smaller than the intrinsic scale-length of the disk, the brightness in the outer regions of decomposed disks at low inclinations will be slightly underestimated, as visible in the relative residual maps (the light yellow region in the left bottom panel of Fig. 3).

At higher inclinations the vertical distribution of stars starts to become visible in the disks, producing isophotal shapes that are rounder than the prediction of the infinitely thin disk fitting functions. In the case of fitting single disks, the consequence is that the fitted exponential will have a larger scale-length than the intrinsic one (see Fig. 2 from Pastrav et al. 2013). In the presence of a bulge, though, this trend is reversed: additional light from the bulge is transferred to the disk, resulting in an exponential fit with a tendentially larger amplitude and a smaller scale-length 
B. A. Pastrav et al.: The effects of dust on the photometric parameters of decomposed disks and bulges
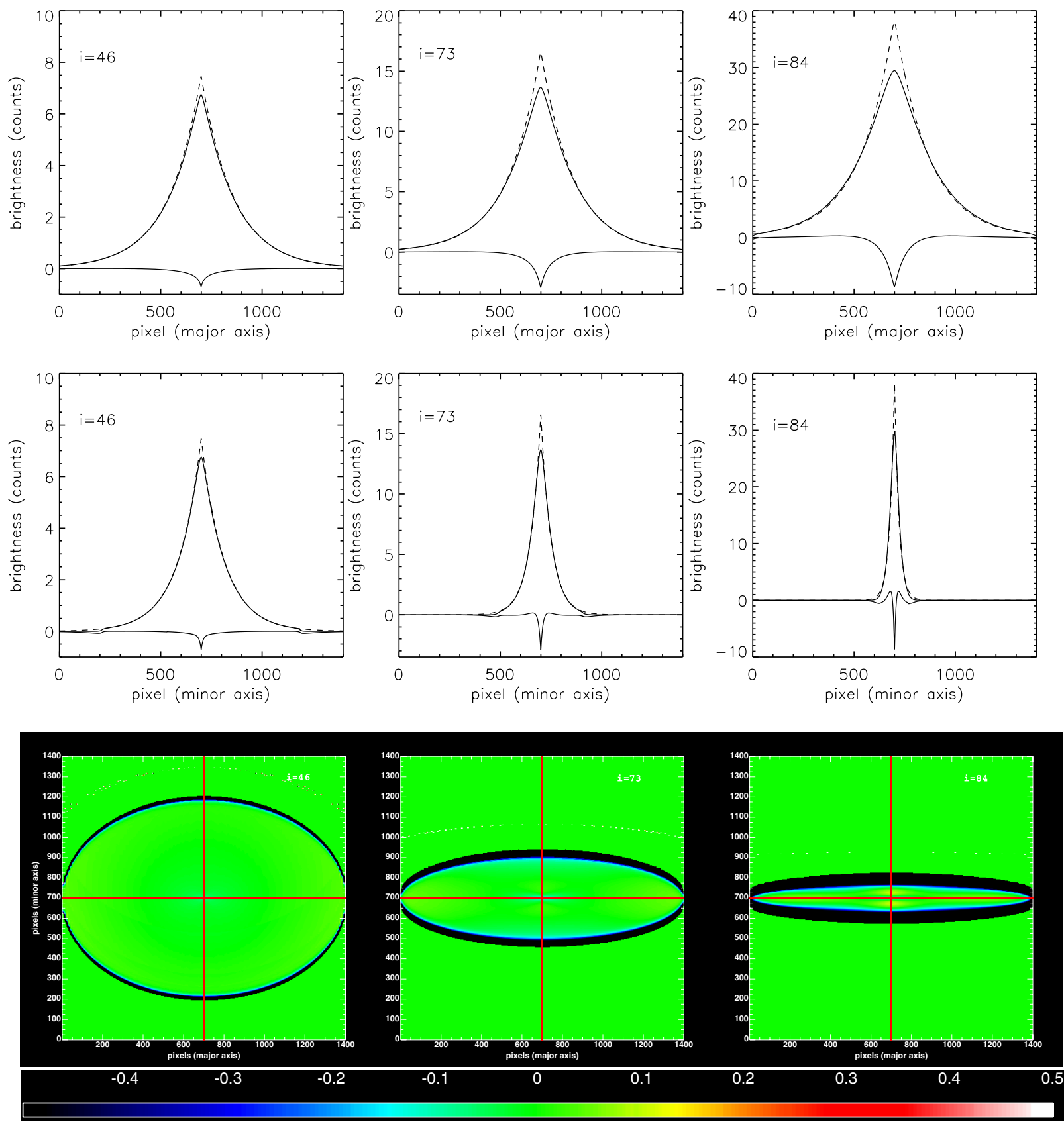

Fig. 3. Major- and minor-axis profiles (upper and middle rows) of simulated dust-free single disks (solid line) and of decomposed disks (dashedline), for $B / D=0.25$, in the $B$-band. Fits are made with an exponential function (for the disk component) and a variable-index Sérsic function (for the exponential bulge). The cuts were taken parallel and perpendicular to the major-axis of the simulated image, through their geometrical centres, at inclinations $1-\cos (i)=0.3,0.7,0.9\left(i=46,73,84\right.$ degrees. Lower row: corresponding relative residuals $\left(\frac{\text { simulation-fit }}{\text { simulation }}\right)$ at the same inclination as the profiles. The red lines show radial and vertical cuts through the geometrical centre of the image.

as the disk is more inclined. As in the case of fitting single disks, the vertical profiles of the fitted disks will fall below the profiles of the simulated image over a certain range of distances from the centre, producing the yellow wings above and below the plane in the relative residual maps (see right bottom panel in Fig. 3).

To quantify these effects we compare the parameters of disks and bulges derived from bulge-disk decomposition of galaxies with $B / D=0.25$ with those obtained from fits to single disks and bulges. The corresponding plots showing the inclination dependence of these projection effects are shown in Figs. 4 and 5.

In Fig. 4 (left) we plot the ratio of the scale-length of decomposed fitted disks to the scale-length of single fitted disks. From the definition of the plotted ratio one can immediately see that the trends from this figure are not directly comparable to those of Fig. 3, since the latter figure shows a comparison with the simulation of a single disk rather than with the fit to a single disk. Thus, to understand the elements of the plotted ratio one needs to take into account both the results from Fig. 3 and the corresponding ones on the fits to single disks (Fig. 2 of Pastrav et al. 2013). As explained above, even at low inclination the scalelength of the fitted decomposed disk is smaller than the intrinsic scale-length of the simulated single disk, and is therefore also smaller than that of the fitted single disks (since at low inclinations the fitted scale-length of single disks recovers very well the intrinsic radial scale-length of the volume stellar emissivity). Since the fitted scale-length of the decomposed disk decreases 

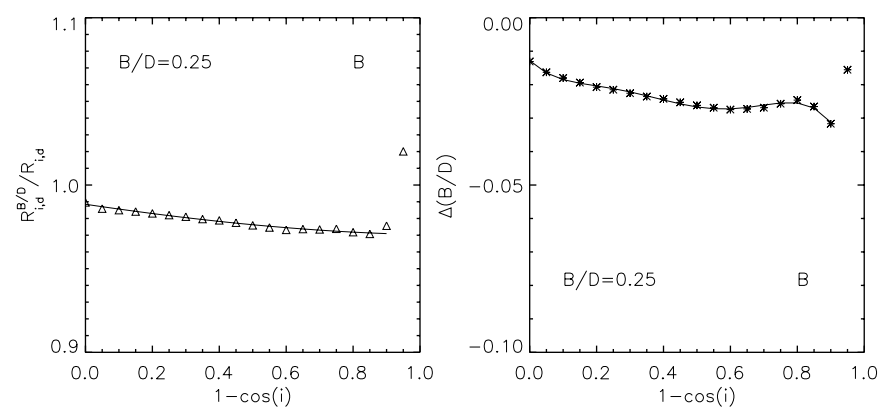

Fig. 4. Left: projection effects $c o r r^{\mathrm{proj}, B / D}$ on the derived scale-length of decomposed disks for $B / D=0.25$. The symbols represent the measurements while the solid lines are polynomial fits to the measurements. The plots represent the ratio between the intrinsic scale-lengths of decomposed and single disks, $R_{i, \mathrm{~d}}^{B / D}$ and $R_{i, \mathrm{~d}}$, respectively, as a function of inclination $(1-\cos (i))$, for the $B$-band. An exponential (disk) plus a variable index Sérsic (bulge) distribution were used for image decomposition. Right: as in the left panel, but for the derived bulge-to-disk ratios, $B / D$. The effects are represented as differences between the intrinsic $B / D$ of decomposed disks and bulges and those of single disks and bulges.
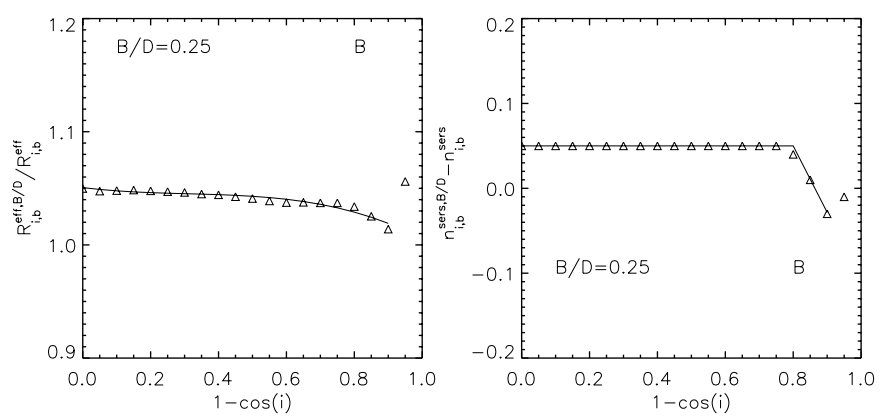

Fig. 5. As in Fig. 4, but for the derived effective radius $R_{i, \mathrm{~b}}^{\mathrm{eff}, B / D}(L e f t)$ and for the derived Sersic indices (Right) of decomposed exponential bulges. The effects on Sérsic indices are represented as differences between the measured Sérsic index of decomposed and single bulges, $n_{i, \mathrm{~b}}^{\text {sers }, B / D}$ and $n_{i, \mathrm{~b}}^{\text {sers }}$, respectively.

with increasing inclination (for low to intermediate inclinations) while the scale-length of the single disk increases with inclination, the ratio of the two decreases. Towards higher inclination both the scale-length of the fitted single and decomposed disks increase, but with the latter having a less stronger increase, resulting in an overall ratio still decreasing with increasing inclination. Although the scale-length is affected by the decomposition, the derived axis-ratio of the decomposed disk is essential identical to that of single disks. Thus, disk axis-ratios are insensitive to projection effects in the decomposition process (in the absence of dust).

In Fig. 5 (left) we plot the ratio of the effective radius of decomposed and single fitted bulges, $R_{i, \mathrm{~b}}^{\mathrm{eff}, B / D}$ and $R_{i, \mathrm{~b}}^{\mathrm{eff}}$. As mentioned before, when bulges are fitted in combination with a disk, there will be a transfer of light from the bulge to the disk, resulting in a underestimation of the light in the centre of the bulge and an overestimation of the effective radius. Projection effects on single bulges also manifest themselves in increasing the measured effective radius with respect to the intrinsic radius of the volume stellar emissivity. When bulge-disk decomposition is performed this overestimation is accentuated. Thus the ratio $R_{i, \mathrm{~b}}^{\mathrm{eff}, B / D} / R_{i, \mathrm{~b}}^{\mathrm{eff}}$ remains supra-unity, with a small decrease when increasing inclination. The derived Sérsic index is $\sim 0.05$ above the value measured on single bulges and remains constant for a large range of inclinations (Fig. 5, right). Since the derived Sérsic index of single bulges was found to be underestimated by more than $\sim 0.05$, the plot in Fig. 5 shows that the measured Sérsic index of decomposed bulges is still lower than the value of 1 (for the exponential bulge).

As expected from the trends described above, the bulge-todisk ratio is slightly underestimated (Fig. 4, right), with the ratio showing a small decrease with increasing inclination.

The projection effects derived for fits with an exponential plus a variable-index Sérsic function are relatively insensitive to the value of $B / D$. Thus, for an increase of the bulge-to-disk ratio to $B / D=0.5$ there is only a $1 \%$ increase in the amplitude of the correction for the scale-length of the exponential disks. Bulges seem to be even less affected (less than $1 \%$ change in the correction), while the overall trends with inclination remain unchanged. Examples of plots showing projection effects on decomposed disks and bulges for galaxies with $B / D=0.5$ are given in Appendix A.

\subsubsection{Fits with two variable-index Sérsic functions}

The second type of fit performed on the two-component simulated dustless galaxies involves fitting a superposition of two variable-index Sérsic functions for the disk and bulge component, respectively. In Fig. 6 we show results from these fits in the form of major- and minor-axis profiles (upper and middle rows) and relative residuals (bottom row) of galaxies with $B / D=0.25$. Comparing these residual maps with those obtained when fitting an exponential plus a variable-index Sérsic function to galaxies having the same bulge-to-disk ratio $B / D=0.25$ (Fig. 2), one can see an overall improvement in the fits at all inclinations. In particular there is an increased area of green colour $(\sim 0 \%$ residuals). The improvement in the reduced $\chi^{2}$ is $12 \%$ at $46^{\circ}, 25 \%$ at $73^{\circ}$ and $87 \%$ at $84^{\circ}$. This is to be expected, due to the additional free parameter of the fit (the Sérsic index of the Sérsic function used to fit the disk).

Although the overall fit is improved, the decomposed components are less accurately extracted, due to the less constrained fit. This can be seen in Fig. 7, where we only show residual maps between the decomposed fitted disks and the simulated single disks (corresponding to Fig. 6) at different inclinations together with the corresponding radial and vertical profiles. In particular one can see that at low inclinations the overestimation of the amplitude in the centre of the disk is accentuated as compared to the situation of an exponential fit to the disk, indicating an even more pronounced transfer of light from the bulge to the disk. The relatively large blue region in the centre of the residual image for $i=46^{\circ}$ (left bottom panel of Fig. 7) represents an overestimation of the surface brightness of around $15 \%$, while the corresponding panel of Fig. 3 only shows a small blue region, with an overestimation of around 5-10\%. Similar conclusions can be drawn from the corresponding profiles, which also show that the brightness of the decomposed disk (plotted as dotted line) exceeds that of the simulated single disk (solid line), in the centre.

At higher inclinations, the yellow wings that were seen above and below the plane in the residual maps of disks decomposed with exponential functions (right bottom panel of Fig. 2) now merge into a region of continuous yellow colour in the centre of the disk, as seen in the right bottom panel of Fig. 7. This means that at high inclinations the surface-brightness in the centre regions of decomposed disks fitted with Sérsic functions will be underestimated by around $15 \%$. Thus, at higher inclinations there is a transfer of light from the disk to the bulge (see also right top and middle panels of Fig. 7). In addition the outer 
B. A. Pastrav et al.: The effects of dust on the photometric parameters of decomposed disks and bulges
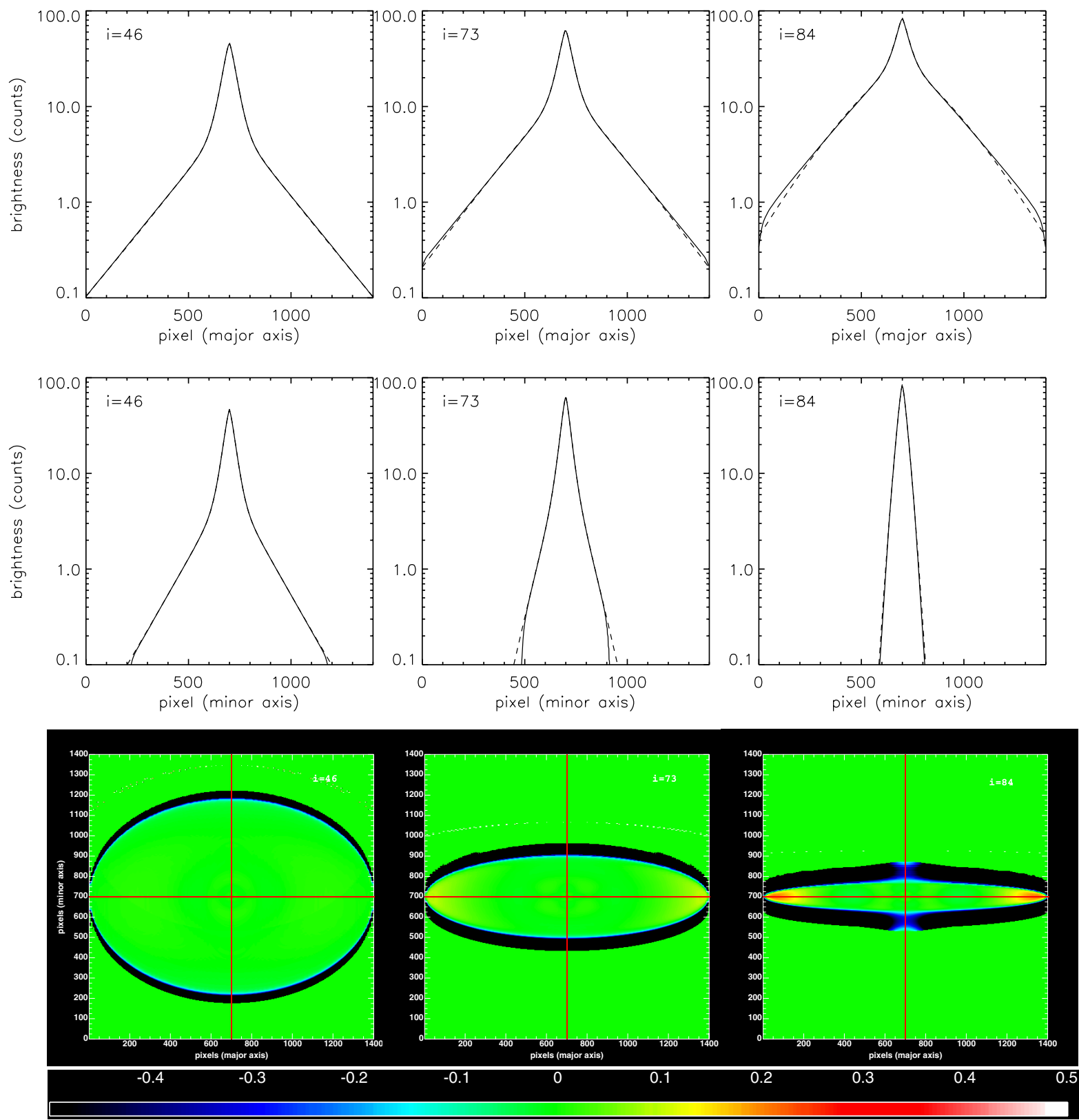

Fig. 6. Major- and minor-axis profiles of dustless galaxies (upper and middle rows) with $B / D=0.25$, in the $B$-band. Fits are made with two variable-index Sérsic functions for the disk and exponential bulge components, respectively. Solid and dashed curves are for simulations and corresponding fits, respectively. The cuts were taken parallel and perpendicular to the major-axis of the simulated image, through the intensity peak, at inclinations $1-\cos (i)=0.3,0.7,0.9\left(i=46^{\circ}, 73^{\circ}, 84^{\circ}\right)$. Lower row: corresponding relative residuals $\left(\frac{\text { simulation-fit }}{\text { simulation }}\right)$, at the same inclination as the profiles. The red lines show radial and vertical cuts through the geometrical centre of the image.

regions of highly inclined decomposed disks fitted with Sérsic functions is less well fitted in comparison with decomposed disks fitted with exponential functions.

To derive the projection effects on the parameters of disks and bulges decomposed from fitting two variable-index Sérsic functions, we compare again the results of the fits with those obtained for single disks and bulges (individually fitted with variable-index Sérsic functions in Pastrav et al. 2013). In Fig. 8 we show the projection effects of decomposed disks, for galaxies with $B / D=0.25$. Close to face-on inclinations the derived effective radius of the decomposed disk is slightly smaller than that derived for single disks, the latter being a good match to the intrinsic effective radius of the volume stellar emissivity. This results in a ratio $R_{i, \mathrm{~d}}^{\mathrm{eff}, B / D} / R_{i, \mathrm{~d}}^{\mathrm{eff}}$ which is slightly less than one at $i=0^{\circ}$ (see Fig. 8 left), similar to the results obtained when performing bulge-disk decomposition with exponential plus Sérsic functions (see Fig. 4, left). With increasing inclination the effective radius of decomposed disks increases, following the transition between an overestimation of the central surface-brightness (light transfer from bulge to disk) to an underestimation of the central surface-brightness (light transfer from disk to bulge). Since the effective radius of single disks fitted with variable Sérsic index functions decreases with increasing inclination (see Fig. 2 from Pastrav et al. 2013), the overall trend of the ratio $R_{i, \mathrm{~d}}^{\mathrm{eff}, B / D} / R_{i, \mathrm{~d}}^{\mathrm{eff}}$ is to increase with increasing inclination.

The slight underestimate in the effective radius of the decomposed disk at low inclinations is also accompanied by a slight overestimate of the derived Sérsic index (which takes the value 

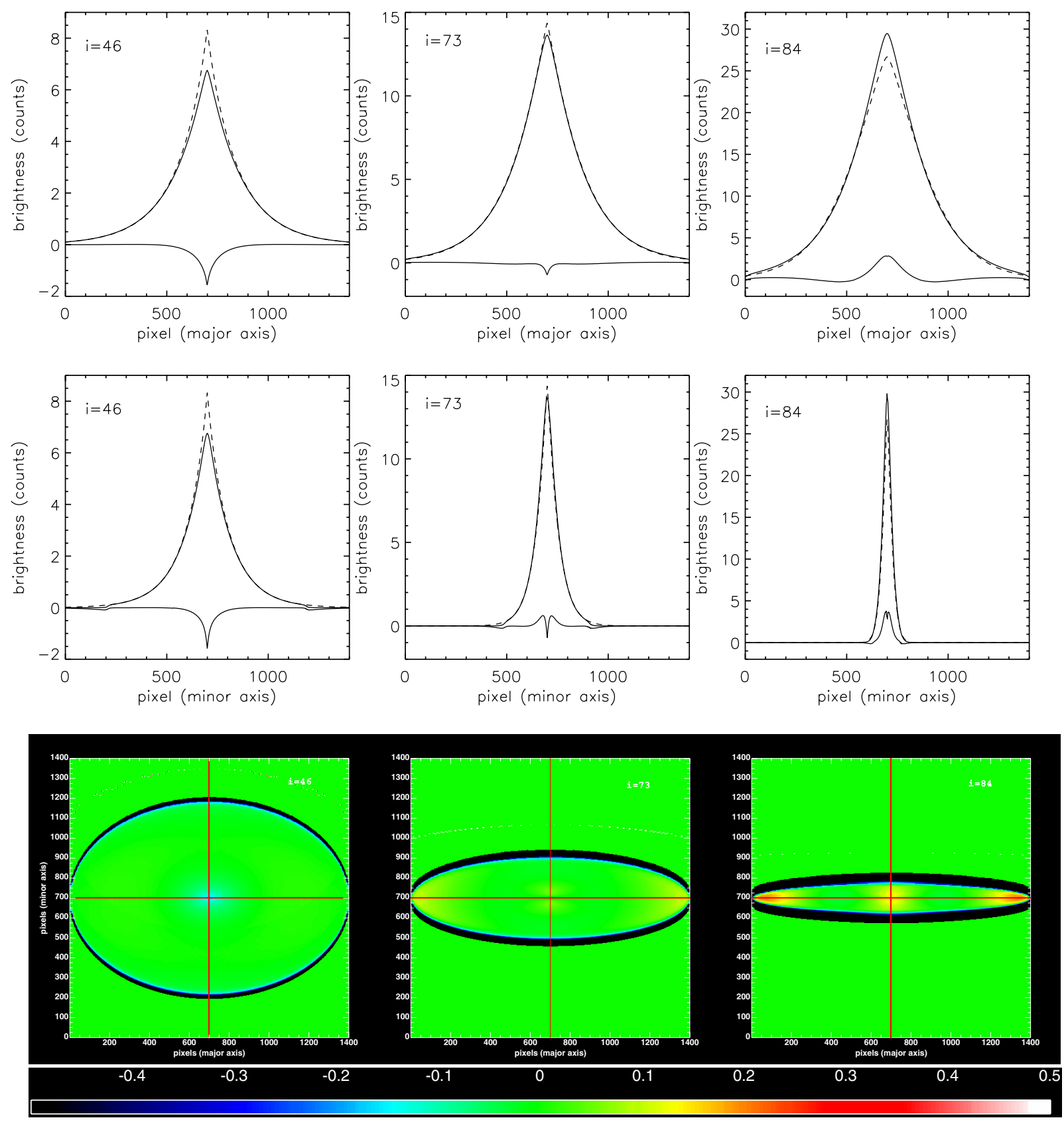

Fig. 7. Major- and minor-axis profiles (upper and middle rows) of simulated dust-free single disks (solid line) and of decomposed disks (dashedline), for $B / D=0.25$, in the $B$-band. Fits are made with two variable-index Sérsic functions (one for disk and one for the exponential bulge component). The cuts were taken parallel and perpendicular to the major-axis of the simulated image, through their geometrical centres, at inclinations $1-\cos (i)=0.3,0.7,0.9\left(i=46,73,84\right.$ degrees. Lower row: corresponding relative residuals $\left(\frac{\text { simulation-fit }}{\text { simulation }}\right)$ at the same inclination as the profiles. The red lines show radial and vertical cuts through the geometrical centre of the image.

of 1.07). Since the fitted Sérsic index of a single disk seen at low inclinations exactly matches the value of 1 (corresponding to an exponential disk), the projection effects manifest in a positive correction for the Sérsic index measured for face-on disks (see right panel of Fig. 8). With increasing inclination, both the derived Sérsic index of single disks and of decomposed disks decreases, but at different rates, such that a decreasing trend in the correction $n_{i, \mathrm{~d}}^{\text {sers, } B / D}-n_{i, \mathrm{~d}}^{\text {sers }}$ is produced.

The projection effects on decomposed bulges are shown in Fig. 9. The effective radius of the decomposed bulge is always larger than that of a single bulge, with the ratio of the two increasing with increasing inclination. The derived Sérsic index is slightly larger than that of a single bulge, and remains essentially constant with increasing inclination.

The bulge-to-disk ratio of decomposed disks and bulges at low inclinations is slightly smaller than the one derived for single components (see Fig. 10). This is to be expected, since, as explained above, light from the bulge is transferred to the disk in the fitting process. This behaviour is similar to the one encountered when doing fits with an exponential plus a Sérsic function, since in both cases the surface-brightness distribution in the central regions of disks is overestimated. At higher inclinations however, an opposite trend is observed, with a bulge-to-disk ratio of decomposed disks and bulges slightly larger than the one 
B. A. Pastrav et al.: The effects of dust on the photometric parameters of decomposed disks and bulges
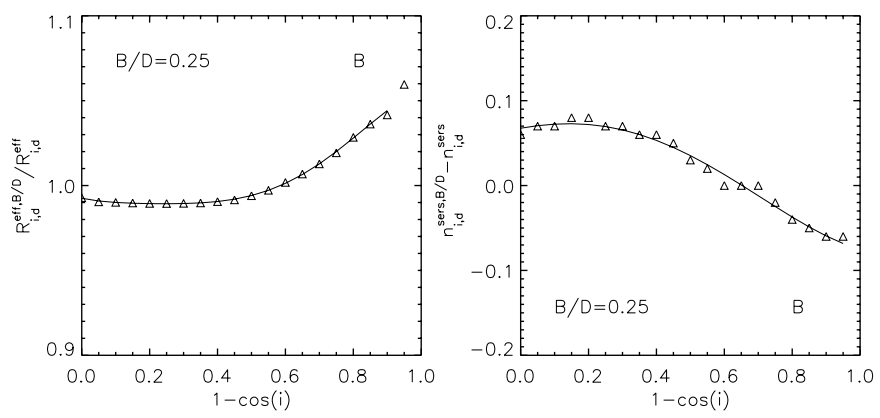

Fig. 8. Left: projection effects $\operatorname{corr}^{\mathrm{proj}, B / D}$ on the derived effective radius of decomposed disks for $B / D=0.25$. The symbols represent the measurements while the solid lines are polynomial fits to the measurements. The plots represent the ratio between the intrinsic effective radius of decomposed and single disks, $R_{i, \mathrm{~d}}^{\mathrm{eff}, B / D}$ and $R_{i, \mathrm{~d}}^{\mathrm{eff}}$, respectively, as a function of inclination $(1-\cos (i))$, for the $B$-band. Two variable index Sérsic functions were used for image decomposition. Right: as in the left panel, but for the derived Sérsic index, $n^{\text {sers }}$. The effects are represented as differences between the measured Sérsic index of decomposed and single disks, $n_{i, \mathrm{~d}}^{\text {sers, } B / D}$ and $n_{i, \mathrm{~d}}^{\text {sers }}$, respectively.
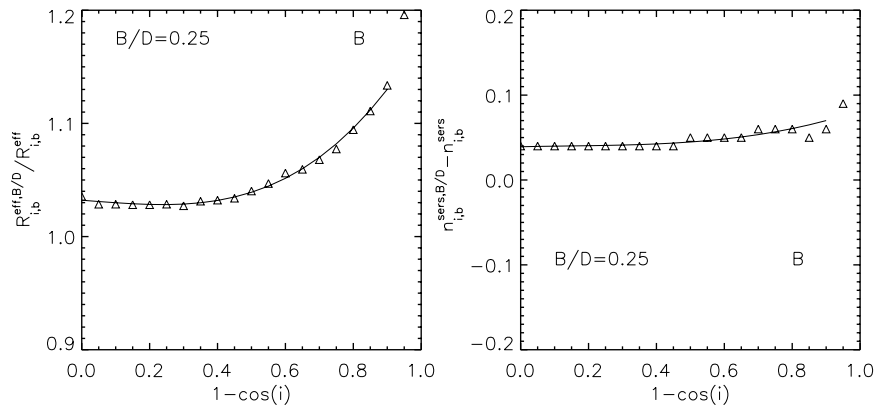

Fig. 9. As in Fig. 8, but for the decomposed exponential bulges.

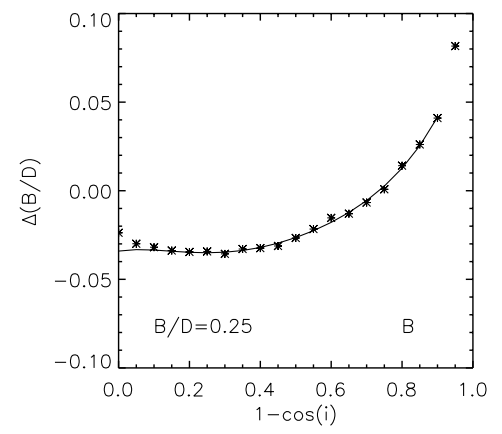

Fig. 10. Projection effects $c o r r^{\text {proj, } B / D}$ on the derived bulge-to-disk ratios, $B / D$. The effects are represented as differences between the intrinsic $B / D$ of decomposed disks and bulges and those of single disks and bulges. Fits are done with two variable Sérsic index functions.

derived from single components. Essentially the correction for projection effects on $B / D$ increases smoothly with inclination. This behaviour is different from the trend obtained in the case of an exponential plus a Sérsic index fit.

The projection effects derived for fits with two variableindex Sérsic function are also relatively insensitive to the value of $B / D$. Thus, for an increase of the bulge-to-disk ratio to $B / D=0.5$ there will only be a $1 \%$ increase in the amplitude of the corrections for the disks. Examples of plots showing projection effects on decomposed disks and bulges for galaxies with $B / D=0.5$ are given in the Appendix A.

\subsection{Galaxies with de Vaucouleurs bulges}

When modelling galaxies containing higher Sérsic index bulges, including de Vaucouleurs bulges, one of the main factors shaping projection effects is the truncation radius of the bulge. Unfortunately this parameter is unknown from observations. From simulations we find that the radial stellar profiles at large galactocentric radii starts to be dominated by the light from the bulge instead of the disk, if galaxies contain bulges with un-truncated stellar distributions. For a galaxy with a de Vaucouleurs bulge, a truncation of the bulge at 3 effective radii is enough to circumvent this problem. For galaxies with higher than $n^{\text {sers }}=4$ the truncation of the bulge would need to be at less than 3 effective radii. Overall the truncation radius would depend in this case on the Sérsic index of the bulge. Whether this has any bearing to reality it is unknown. Overall this pins down to the underlying problem that we do not know what the intrinsic distribution of the volume emissivity of the bulge is, and that there is no physical interpretation attached to the Sérsic distribution that is used to described the projected stellar distribution (images) of bulges. The deprojected Sérsic distribution does not have an exact analytic formula due to the singularity in the centre, and therefore approximate formulae have been proposed to describe the volume stellar emissivity. In our model we consider an analytic formula that, when integrated to infinity reproduces the Sérsic distribution of a 2D map. Nonetheless, if bulges are truncated, and we insist in preserving the same analytic formulation, we end up in simulations that are not perfectly fitted by Sérsic distributions ${ }^{1}$. We included these deviations in our projection effects, although, unlike the case of the disk, this is a reverse problem to the disk: in disks we know the intrinsic volume emissivity and therefore we can predict and compare with observations the projected distribution, while in bulges we know the projected stellar emissivity, but we cannot exactly predict the intrinsic stellar emissivity, and therefore we cannot directly compare with observations. Unlike the disk, it is therefore unclear whether what we call projection effects on bulges is a real effect or just a limitation of our knowledge of the true 3D stellar distribution of bulges. In composite systems with disk and bulges, the combined projection effects of disk and bulges that act on the bulge-disk decomposition amplifies if the truncation radius of the bulge is short, as is the case for de Vaucouleurs bulges in our model. This produces projection effects that are larger than in the case of exponential bulges, which we truncated at 10 effective radii. We caution therefore the reader that corrections for projection effects derived for systems with de Vaucouleurs bulges are less certain.

Following the same procedure as in Sect. 5.1, we derive corrections for projection effects both for decompositions involving an exponential plus a variable index Sérsic function and fits with two variable index Sérsic functions. Examples of corresponding plots with corrections are given in Appendix B.

\section{The effects of dust on the bulge-disk decomposition}

In this section we present and discuss the effects of dust on the process of decomposing galaxy images and therefore on the photometric parameters of decomposed disks and bulges. As mentioned before, unlike the corrections measured on single components, it is not possible to only measure a dust effect on the

1 As shown in Pastrav et al. (2013), the shorter the truncation radius is, the larger the deviation from the Sérsic distribution. 

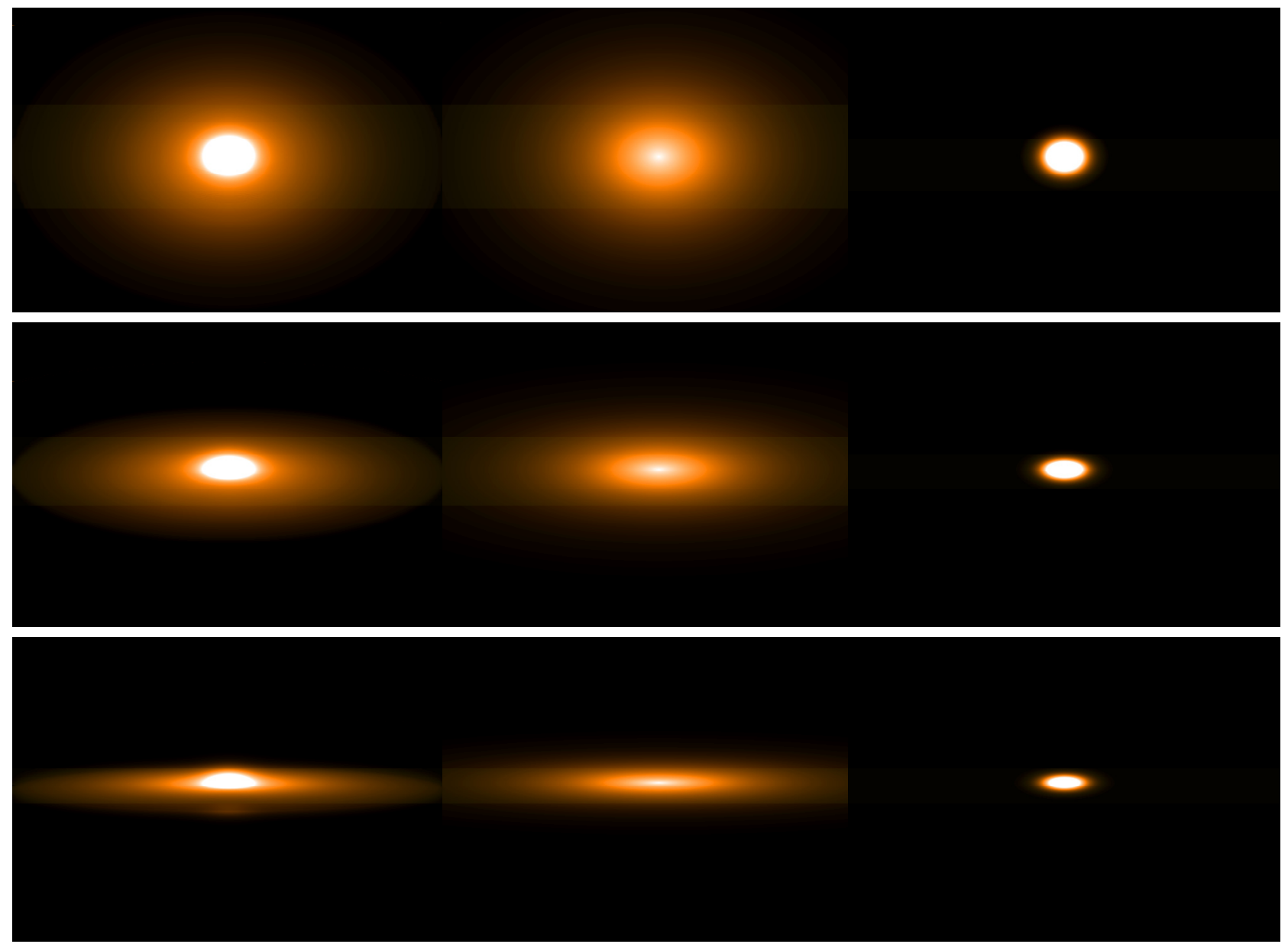

Fig. 11. Simulated images of galaxies with exponential bulges and $B / D=0.25$ (left column) and corresponding decomposed disks and bulges (middle and right columns). The bulge-disk decomposition fit was made with an exponential plus a variable index Sérsic function, at inclinations $1-\cos (i)=0.3,0.7,0.9\left(i=46^{\circ}\right.$ (first row), $73^{\circ}$ (second row) and $84^{\circ}$ (third row)), for $\tau_{B}^{f}=4.0$.

decomposition. The measurements are for the joint effect of dust and projections on the decomposition. Thus, using Eqs. (3)-(7), we relate the measured photometric parameters of decomposed disks and bulges to those obtained in our previous study (the apparent values from fitting individual components) in order to quantify $\operatorname{corr}^{B / D}$, the dust and projections effects on the bulgedisk decomposition. Then, by subtracting the corrections for projection effects (as described in Sect. 5), we can isolate the pure dust effects, corr $^{\text {dust, } B / D}$. These effects are quantified for galaxies with exponential bulges (Sect. 6.1) and de Vaucouleurs bulges (Sect. 6.2)

One of the main problems when performing bulge-disk decomposition of dusty galaxies is the dust-induced asymmetries in the surface-brightness distributions, in particular at higher inclinations. These asymmetries are present in both the dustattenuated disk and bulge, as described in Pastrav et al. (2013), and because of them, the position of the intensity peak does not coincide with the geometrical centre of the image. In addition, the position of the peak intensity of each dust-attenuated component is differently shifted from the geometrical centre. Therefore the combined image will have a peak intensity which will coincide neither with the geometrical centre, nor with the true position of the peak intensity of either disk or bulge. As a consequence, the resulting bulges and disks will be imperfectly subtracted when performing bulge-disk decomposition with simple analytic templates, irrespective of the combination of functions used to fit the composite systems (exponential plus Sérsic or Sérsic plus Sérsic).

\subsection{Galaxies with exponential bulges}

\subsubsection{Fits with exponential + variable-index Sérsic functions}

The first type of fit performed on the two-component simulated galaxies consists of an exponential plus a variable-index Sérsic function for the disk and bulge component, respectively. Examples of bulge-disk decompositions performed in this way are given in Fig. 11. In Fig. 12 we also show results from these fits in the form of major- and minor-axis profiles (upper and middle rows) and relative residuals (bottom row). One can see the afore-mentioned asymmetries about the major-axis, which increase with increasing inclination of the disk. The blue region of negative residuals in the outer disk seen in Fig. 12 is due to the fact that the simulations are truncated while the fits extend to infinity.

Another effect which influences the decomposition is the flattening of the radial profiles in the inner regions of dustattenuated disks, in particular for higher values of dust opacity, as already discussed in Pastrav et al. (2013). When such disks exist in isolation (without a bulge) and are fitted with an exponential function, the depression of the surface-brightness in the centre of disks results in a fit with an exponential model 
B. A. Pastrav et al.: The effects of dust on the photometric parameters of decomposed disks and bulges
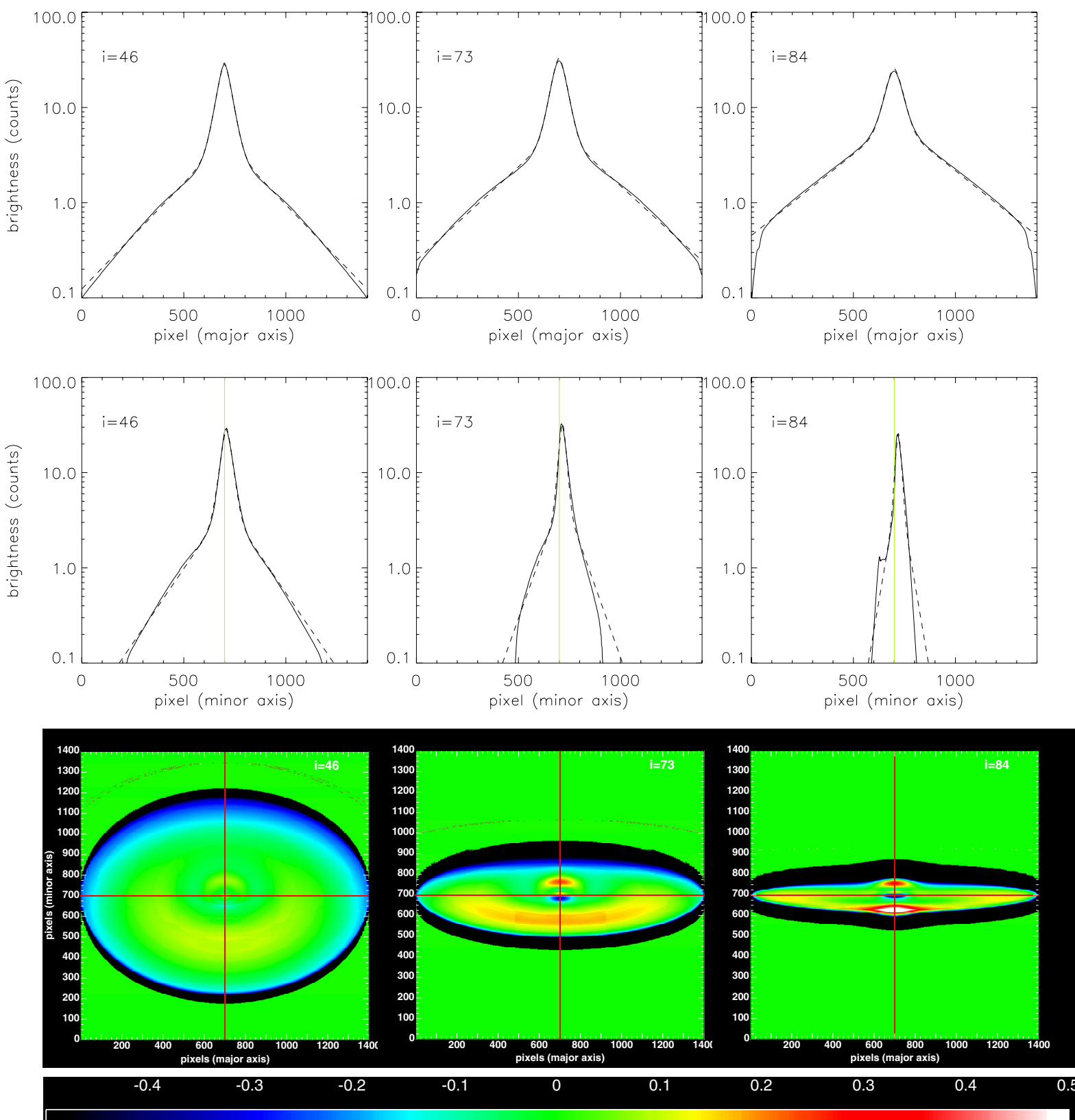

$-0.1$

0

0.1

0.2

0.3

0.4

Fig. 12. Major- and minor-axis profiles of dusty galaxies (upper and middle rows) with $B / D=0.25$, and $\tau_{B}^{f}=4.0$, in the $B$-band. Fits are made with an exponential function (for the $\mathrm{f}$ disk component) and a variable-index Sérsic function (for the exponential bulge). Solid and dashed curves are for simulations and corresponding fits, respectively. The cuts were taken parallel and perpendicular to the major-axis of the simulated image, through the intensity peak, at inclinations $1-\cos (i)=0.3,0.7,0.9\left(i=46^{\circ}, 73^{\circ}, 84^{\circ}\right)$. The light green line shows a cut through the geometrical centre of the image. Lower row: corresponding relative residuals $\left(\frac{\text { simulation-fit }}{\text { simulation }}\right)$, at the same inclination and opacity as the profiles. The red lines show radial and vertical cuts through the geometrical centre of the image.

having a larger scale-length than the intrinsic one. However, in the presence of a bulge, the flattening of the disk profile in the centre is wrongly compensated for by the fitting routine with stellar light from the bulge. This can be seen in Fig. 13, where we plot examples of relative residuals between simulated single dusty disks and corresponding decomposed disks. The blue region in the centre (for $i=46^{\circ}$ and $73^{\circ}$ ) is due to the exponential form of the decomposed disk which rises above the flattened central region of the simulated attenuated single disk. At lower dust opacities, when the flattening of the disk is small and happens within one effective radius of the bulge, the routine will transfer enough light from the bulge to reasonably compensate for the flattening of the disk. Therefore the derived scale-length is closer (or slightly smaller) to the intrinsic scale-length of the disk (measured at the same inclination in the absence of dust). At higher optical depth though, when the disk is optically thick until large radii, beyond the effective radius of the bulge, there is still a transfer of light from the bulge to the disk, but not enough to compensate for the more pronounced flattening. Therefore, to account for the remaining depression in the surface-brightness, the routine will tend to overestimate the scale-length of the decomposed disk (with respect to the dustless case), as in the case of a single disk analysis. However, the overestimation will be smaller than in the case of a single disk. To conclude, the derived scale-length of a decomposed disk is close to the intrinsic one at smaller opacities and is overestimated at higher opacities. 

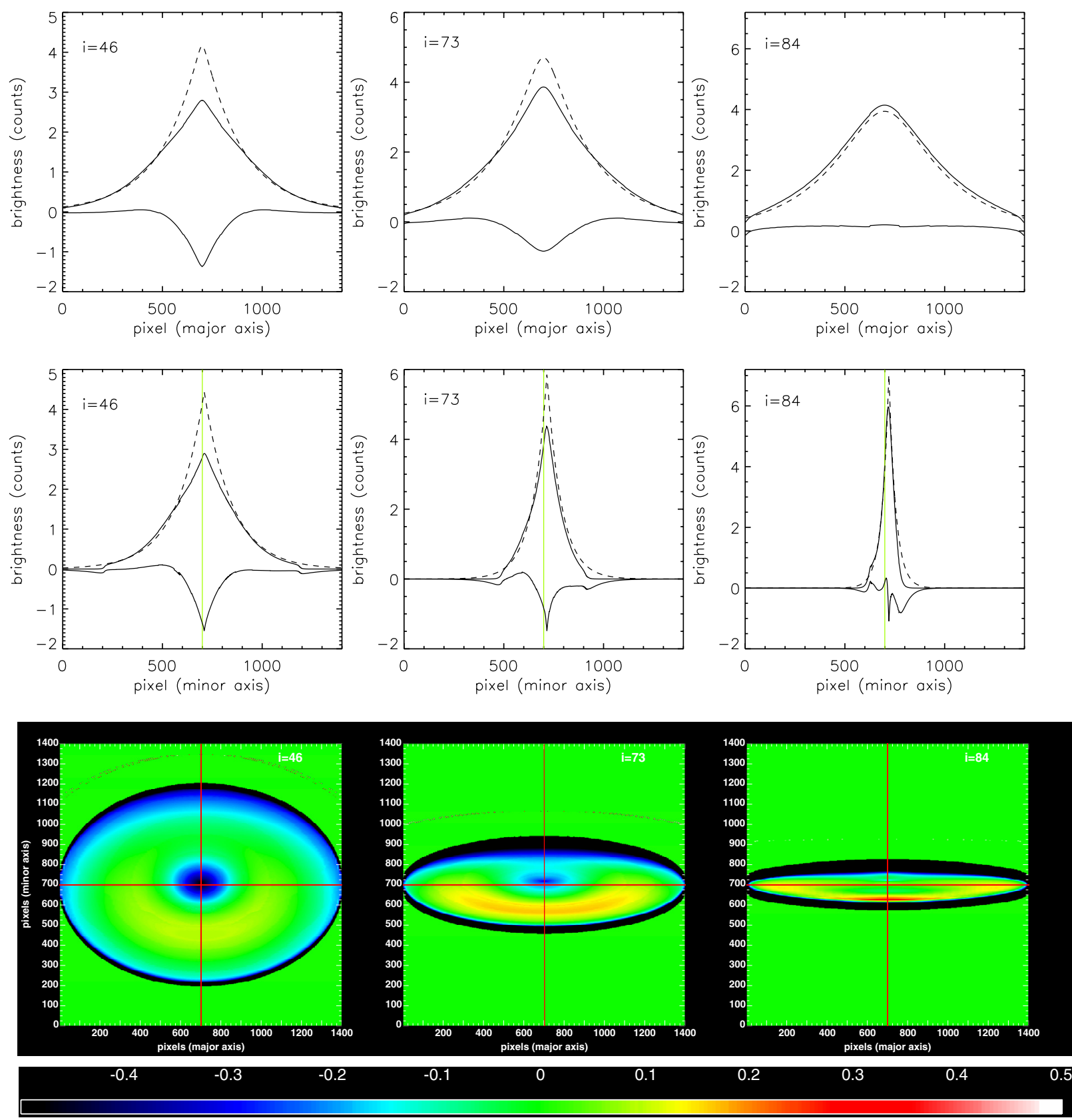

Fig. 13. Major- and minor-axis profiles (upper and middle rows) of simulated dusty single disks (solid line) and of decomposed dusty disks (dashed-line), for $B / D=0.25$ and $\tau_{B}^{f}=4.0$, in the $B$-band. Fits are made with an exponential function (for the disk component) and a variableindex Sérsic function (for the exponential bulge). The cuts were taken parallel and perpendicular to the major-axis of the simulated image, through their geometrical centres, at inclinations $1-\cos (i)=0.3,0.7,0.9(i=46,73,84$ degrees. The light green line shows a cut through the geometrical centre of the image. Lower row: corresponding relative residuals $\left(\frac{\text { simulation-fit }}{\text { simulation }}\right)$, at the same inclination and opacity as the profiles. The red lines show radial and vertical cuts through the geometrical centre of the image.

We note here that this effect is not visible in Fig. 13, since the outer regions of the disks in the residual maps are dominated by the difference between the truncated simulation and the untruncated model. However, in all cases the derived scale-lengths of decomposed disks will be smaller than the derived scale-lengths in the absence of a bulge (see Fig. 14).

Conversely, the decomposed bulge will have a slightly flatter profile in the centre than in reality, since light from the simulated bulge has been transferred to the simulated disk, resulting in a fit with a smaller Sérsic index than in the case of a pure attenuated bulge (see Fig. 15). As expected, the corrections $\operatorname{corr}^{\text {dust, } B / D}$ are larger in the $B$-band than in the $K$-band. The derived effective radius of bulges is smaller than that of single attenuated bulges, (see Fig. 16).

Since in the decomposition process light from the bulge is transferred to the disk, in particular for higher opacity, the derived bulge-to-disk ratio will be smaller than the bulge-to-disk ratio of a single bulge and a single disk attenuated by the same dust opacity. This can be seen in Fig. 17, where the dust correction takes negative values.

The results from Figs. 14 to 17 are for a $B / D=0.25$. The same analysis performed on simulations having $B / D=$ 0.5 show very little differences in the results (see examples in Appendix C). For disks the amplitude of the effects slightly 
B. A. Pastrav et al.: The effects of dust on the photometric parameters of decomposed disks and bulges

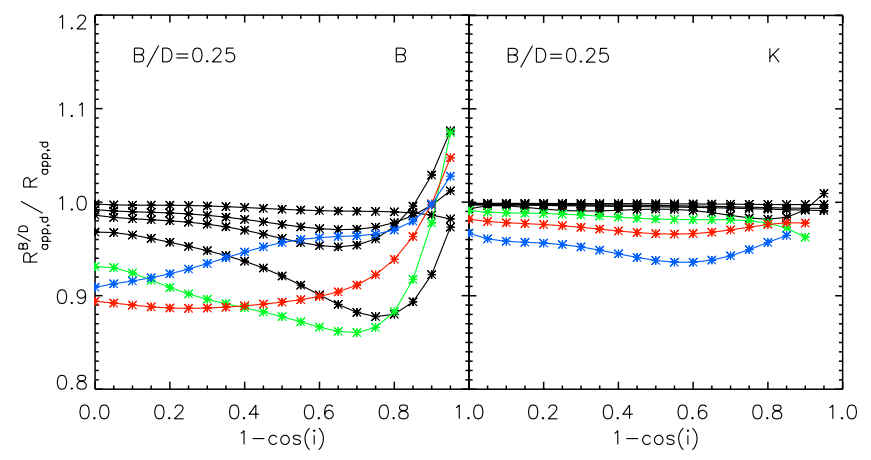

Fig. 14. Dust effects $\left(\operatorname{corr}^{\text {dust, } B / D}\right)$ on the derived scale-length of decomposed disks for $B / D=0.25$. The solid lines are polynomial fits to the measurements. The plots represent the ratio between the apparent scalelengths of decomposed and single disks, $R_{\text {app,d }}^{B / D}$ and $R_{\text {app,d }}$, respectively (corrected for projection effects), as a function of inclination $(1-\cos (i)$ ), for the $B$ and $K$ optical bands. An exponential (disk) plus a variable index Sérsic (bulge) distribution were used for image decomposition. The black curves are plotted for $\tau_{B}^{f}=0.1,0.3,0.5,1.0$, while the other curves correspond to $\tau_{B}^{f}=2.0$ (green), 4.0 (red) and 8.0 (blue).

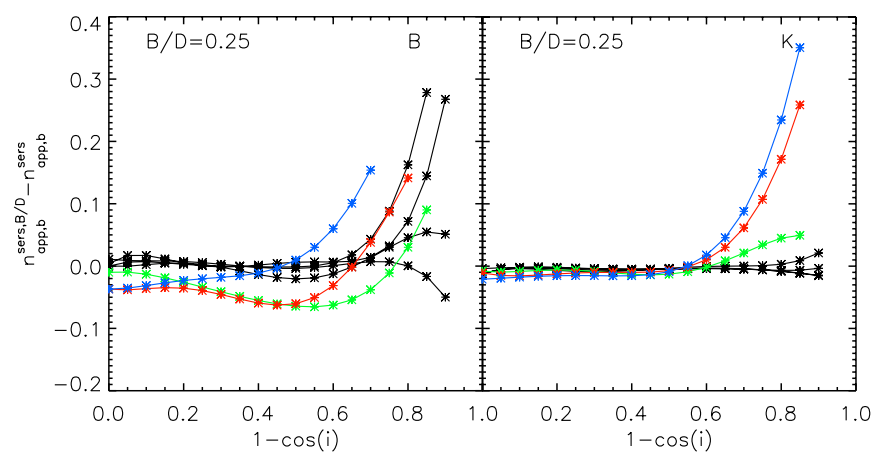

Fig. 15. As in Fig. 14, but for the derived Sérsic index of decomposed exponential bulges. The effects are represented as differences between the derived Sérsic index of decomposed and single bulges, $n_{\text {app,b }}^{\text {sers, } B / D}$ and $n_{\mathrm{app}, \mathrm{b}}^{\mathrm{sers}}$, respectively.

increases with increasing $B / D$. For bulges the amplitude of the effects decreases with increasing $B / D$. The trends with inclinations remain the same.

\subsubsection{Fits with two variable-index Sérsic functions}

For bulge-disk decomposition performed with two variableindex Sérsic functions there is an extra free parameter for fitting the disk component, namely the Sérsic index of the disk. This results in an overall better fit for the composed system. In addition, unlike the dustless case, the decomposed disk and bulge are also better fitted in this way, with a less transfer of light from the bulge to the disk, resulting in a solution which is closer to the single disk and single bulge cases. This is because dust flattens the Sérsic profiles in the centre of bulges and disks, making the decomposition less ambiguous and degenerate than in the dust free case, at least for the low and intermediate inclination cases. As shown in Pastrav et al. (2013), the flattening of the central parts of single disks due to attenuation is fitted with a Sérsic index having a lower value than the intrinsic one. When a bulge is also present, GALFIT will find a solution with a slightly larger Sérsic index than for the single disk (see Fig. 18), because light transfer from the bulge still occurs for all opacities. Because of

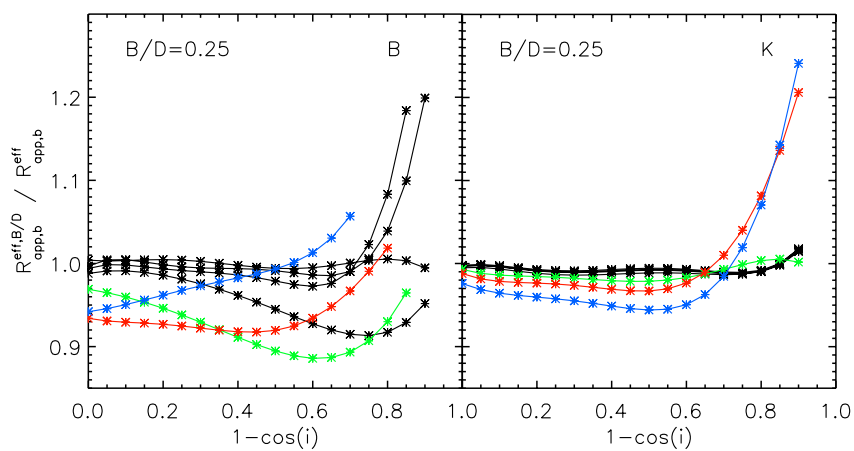

Fig. 16. As in Fig. 14, but for the derived effective radius of decomposed exponential bulges.

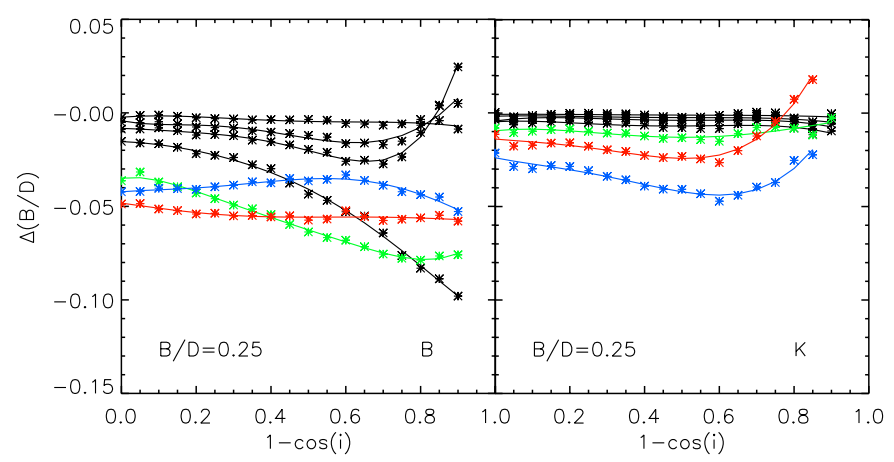

Fig. 17. Dust effects $\left(c o r r^{\text {dust, } B / D}\right)$ on the derived bulge-to-disk ratios, $B / D$. The effects are represented as differences between the apparent $B / D$ of decomposed disks and bulges as those of single disks and bulges. An exponential (disk) plus a variable index Sérsic (bulge) distribution were used for image decomposition. The curves are plotted for $\tau_{B}^{f}=0.1,0.3,0.5,1.0$ (from the top towards the bottom), while the other curves correspond to $\tau_{B}^{f}=2.0$ (green), 4.0 (red) and 8.0 (blue).

this the derived effective radii will be close (or slightly smaller) to the ones derived for single disks, as shown in Fig. 19.

The effective radii (see Fig. 21) and the derived Sérsic index (see Fig. 20) of the bulge is relatively insensitive to the existence of a disk, meaning the solution is very close to that derived for single bulges, at least for inclinations less than $1-\cos (i)=0.6$.

The derived bulge-to-disk ratio is very close to the one obtained for single bulges and disks, for a large range of inclinations and opacities (see Fig. 22). Only at large optical depth and does the bulge-to-disk ratio decrease with respect to single components case. In the $K$ band the corrections are negligible.

The results presented in Figs. 18 to 21 are for $B / D=0.25$. A similar analysis performed on simulations made with $B / D=$ 0.50 shows that a more prominent bulge does not significantly change the results for corr $^{\text {dust, } B / D}$ (see examples in Appendix C). As in the case of fits done with exponential plus Sérsic functions, the amplitude of the effects for disks increases with increasing $B / D$ and decreases for bulges. Thus, irrespectively of the fitting functions (exponential plus Sérsic or Sérsic plus Sérsic) bulgedisk decompositions of systems containing exponential bulges are only a slow varying function of the bulge-to-disk ratio.

\subsection{Galaxies with de Vaucouleurs bulges}

In the case of de Vaucouleurs bulges the overall trends are the same as those for exponential bulges, but with the amplitude of $\operatorname{corr}^{\text {dust, } B / D}$ being larger for any given inclination and opacity. This means that for higher Sérsic indices the decomposition 


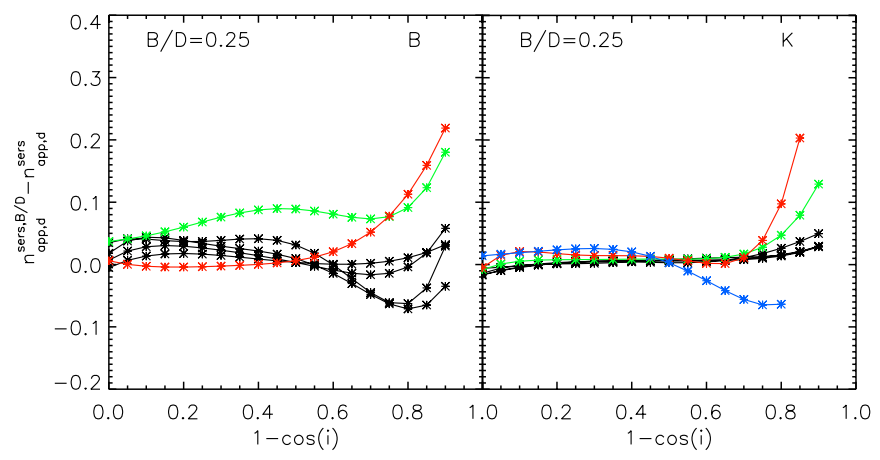

Fig. 18. Dust effects $\left(\operatorname{corr}^{\text {dust, } B / D}\right)$ on the derived Sérsic index of decomposed disks, for $B / D=0.25$. The solid lines are polynomial fits to the measurements. The plots represent the difference between the derived Sérsic index of decomposed and single disks, $n_{\mathrm{app}, \mathrm{d}}^{\mathrm{sers} B} / D$ and $n_{\mathrm{app}, \mathrm{d}}^{\mathrm{sers}}$, respectively (corrected for projection effects), as a function of inclination $(1-\cos (i))$, for the $B$ and $K$ optical bands. Two variable Sérsic index functions were used for image decomposition. The black curves are plotted for $\tau_{B}^{f}=0.1,0.3,0.5,1.0$, while the other curves correspond to $\tau_{B}^{f}=2.0$ (green), 4.0 (red) and 8.0 (blue).

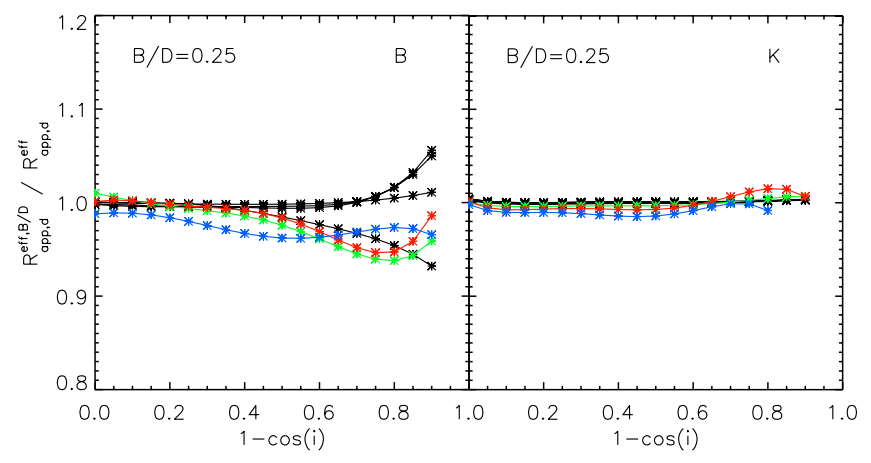

Fig. 19. As in Fig. 18, but for the derived effective radii of decomposed disks.

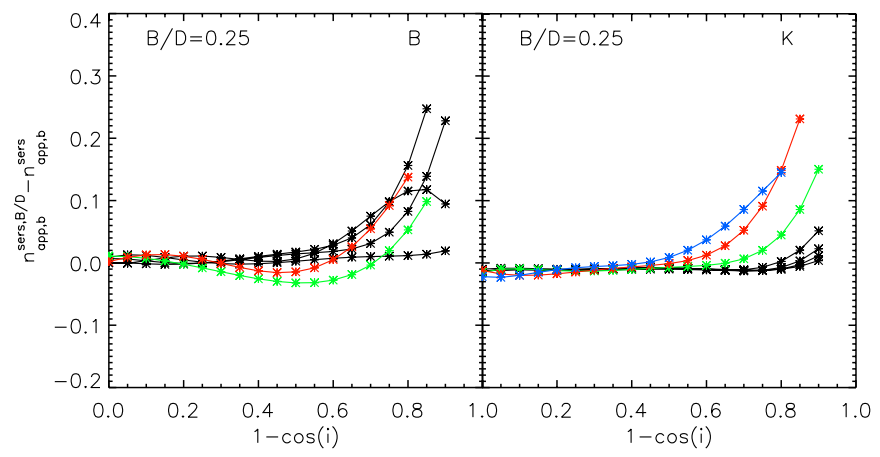

Fig. 20. As in Fig. 18, but for the derived Sérsic index of decomposed exponential bulges. The effects are represented as differences between the derived Sérsic index of decomposed and single bulges, $n_{\mathrm{app}, \mathrm{b}}^{\mathrm{sers} B}$ and $n_{\mathrm{app}, \mathrm{b}}^{\mathrm{sers}}$, respectively.

between disk and bulge starts to be biased. Examples of plots with the corrections are shown in Appendix D.

\section{Single Sérsic fits}

This part of our study is motivated by the fact that single Sérsic fits are commonly used in image analysis (e.g. Hoyos et al. 2011; Simard et al. 2011; Kelvin et al. 2012; Lackner \& Gunn 2012; Bruce et al. 2012; Bernardi et al. 2012; Häußler et al. 2013).

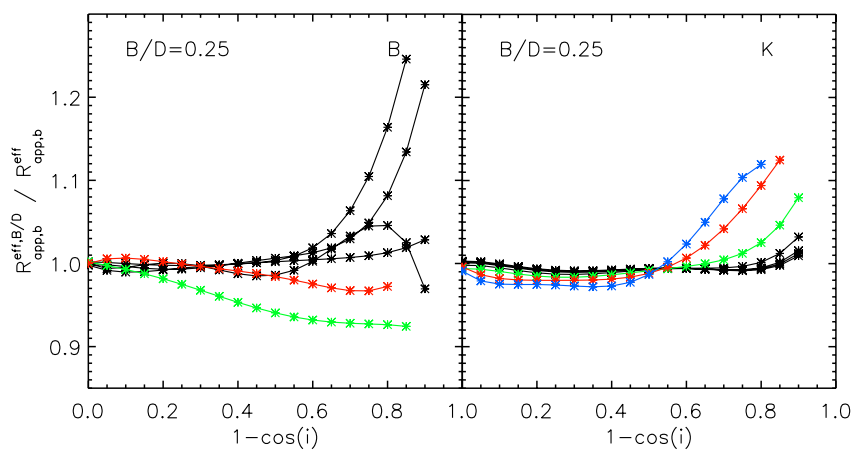

Fig. 21. As in Fig. 18, but for the derived effective radius of decomposed exponential bulges.

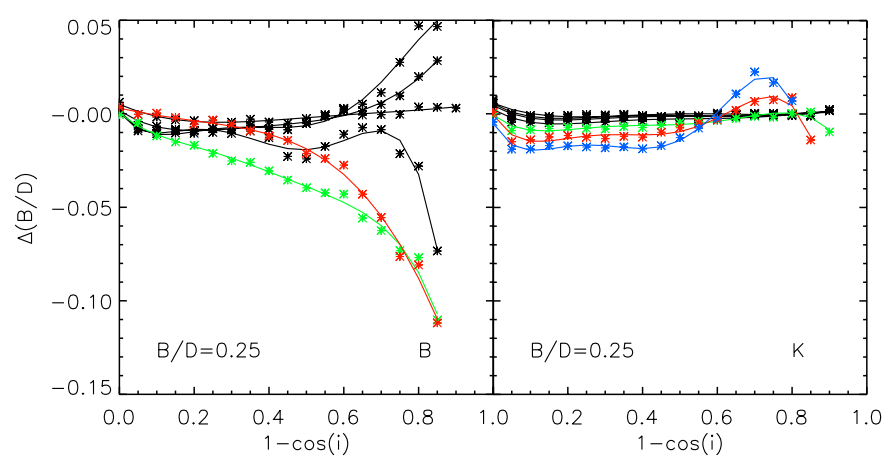

Fig. 22. Dust effects $\left(\operatorname{corr}^{\text {dust, } B / D}\right)$ on the derived bulge-to-disk ratios, $B / D$. The effects are represented as differences between the apparent $B / D$ of decomposed disks and bulges as those of single disks and bulges. Two variable Sérsic index functions were used for image decomposition. The black curves are plotted for $\tau_{B}^{f}=0.1,0.3,0.5,1.0$, while the other curves correspond to $\tau_{B}^{f}=2.0$ (green), 4.0 (red) and 8.0 (blue).

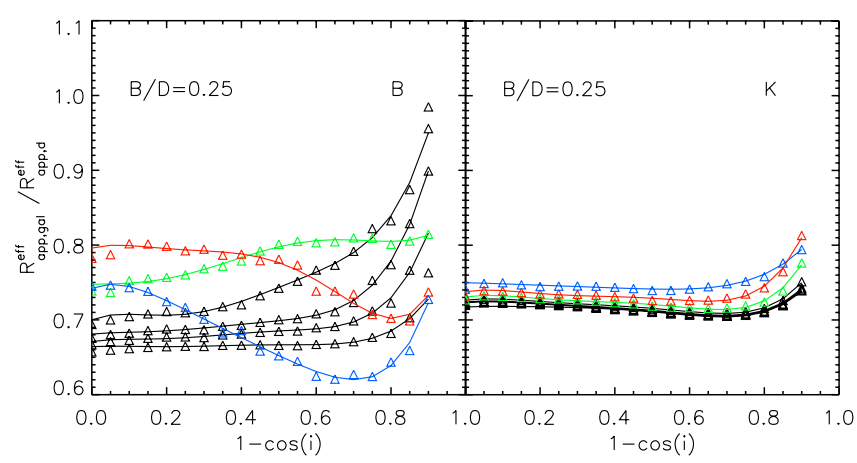

Fig. 23. Dust effects $c o r r^{s S}$ on the derived effective radius of galaxies fitted with single Sersic functions. The symbols represent the measurements while the solid lines are polynomial fits to the measurements. The plots represent the ratio between the effective radius of a bulge+disk system and a single disk, $R_{\text {app }}^{\mathrm{sS}}$ and $R_{\text {app,d }}$ respectively, as a function of inclination $(1-\cos (i))$, for the $B$ and $K$ optical bands. The black curves are plotted for $\tau_{B}^{f}=0.1,0.3,0.5,1.0$ (from the bottom towards the top), while the other curves correspond to $\tau_{B}^{f}=2.0$ (green), 4.0 (red) and 8.0 (blue).

This is usually done for large sample of galaxies with marginal resolution, where morphological components cannot be clearly separated/distinguished, or where a two-component fit is not a significant improvement over a single Sérsic fit.

We show here that the derived effective radius of a composite galaxy fitted with single Sérsic functions is strongly underestimated. This can be seen in Fig. 23, where the effect is visible for 
both the $B$ and the $K$ band. The strongest effect appears for the optically thinner cases, where the bulge is biasing the general solution of the fit. For galaxies with higher optical depth the attenuation due to dust is flattening the profiles in the centre of the galaxy, making the effect of bulges less pronounced, and therefore bringing the results of single Sérsic fits closer to the real size of the disk. The effects strongly depend on the $B / D$ parameter, with higher values of $B / D$ resulting in a stronger underestimation of galaxy sizes, for the same inclination and dust opacity.

\section{Application: the inclination dependence of dust effects}

One important application of our modelling is the prediction for the inclination dependence of the effects of dust on the derived scale-lengths of disks. To compare our predictions with observations we used the photometric data derived by Simard et al. (2011) for galaxies from the Legacy area of the Sloan Digital Sky Survey (SDSS) Data Release 7. In total Simard et al. performed bulge-disk decompositions in $g$ and $r$ bands for 1123718 galaxies using three different type of fits: an exponential disk plus a de Vaucouleurs bulge, an exponential disk plus a Sérsic bulge and a single Sérsic fit. We used the measurements in $r$ band for exponential scale-lengths derived from fits with an exponential disk plus a Sérsic bulge. From these we selected only the measurements for which these fits represent a significant improvement over a single Sérsic fit, as listed by Simard et al. We also selected galaxies with redshifts $z \leq 0.08$. This gave us a sample of 117833 galaxies. From this we further selected galaxies with $B / D<0.35$. This criterion was applied to ensure a higher probability of selecting a sample of bona fide spiral disks. This left us with a sample of 38555 galaxies with measured exponential disk sizes, integrated magnitudes and inclinations. Since the inclinations listed in Simard et al. (2011) are not corrected for projection effects (due to the vertical distribution of stars), we re-calculated these by applying the corrections corr $^{\text {proj }}$ from our model, as listed in Pastrav et al. (2013). In Fig. 24 we show the size-luminosity relation for our sample, as plotted with black stars. A well defined correlation can be seen, with more luminous galaxies having larger sizes. The sharp upper bound of the distribution is almost certainly due to the surface-brightness limit of the photometric imaging SDSS survey. We also plotted as red crosses the data corresponding to galaxies with disk inclinations $1-\cos (i)>0.8$. It is interesting to see that the red points occupy only the brighter part of the correlation, with most of the points having disk magnitudes brighter than -17 . No red points exist for the very faint end of the correlation. This suggests that galaxies with the smaller axis-ratios are biased towards more luminous galaxies, due to the fact that low luminosity galaxies with edge-on orientations are missed in the flux limited survey, which may be plausibly attributed to the larger attenuation by dust of edge-on galaxies. We made similar tests for the other bins in inclinations, where we found no bias. Because of this we excluded the galaxies with $1-\cos (i)>0.8$ and we only compared the prediction of our model with data for inclinations in the range $1-\cos (i)<0.8$. This left us with a sample of 33770 galaxies.

To compare our model predictions with the data we derived the average exponential scale-length for each bin in inclination, where the bins were taken to be $\Delta \cos (i)=0.05$. For the model predictions we considered the whole chain of corrections

$\operatorname{corr}\left(R_{\mathrm{d}}\right)=\operatorname{corr}^{\mathrm{proj}}\left(R_{\mathrm{d}}\right) * \operatorname{corr}^{\mathrm{dust}}\left(R_{\mathrm{d}}\right) * \operatorname{corr}^{B / D}\left(R_{\mathrm{d}}\right)$

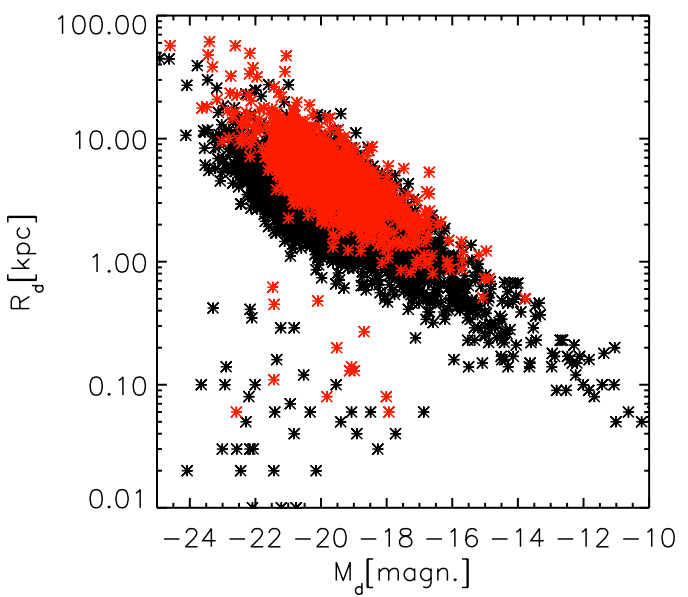

Fig. 24. Disk size-luminosity relation for a sample of galaxies selected from Simard et al. (2011). Galaxies with inclinations $1-\cos (i)>0.8$ are overplotted as red crosses.

where $R_{\mathrm{d}}$ is the exponential (radial) scale-length of the stellar disk, $\operatorname{corr}^{\mathrm{proj}}\left(R_{\mathrm{d}}\right)$ are the projection effects listed in Pastrav et al. (2013), $\operatorname{corr}^{\text {dust }}\left(R_{\mathrm{d}}\right)$ are the effects of dust on the scale-length of disks seen in isolation, as listed in Pastrav et al. (2013), and $\operatorname{corr}^{B / D}\left(R_{\mathrm{d}}\right)$ are the effects of dust on the scale-length of disks seen in combination with a bulge, as derived in this paper. As in Pastrav et al. (2013), the corrections for an average population of spiral galaxies were calculated for $\tau_{B}^{f}=4$. The choice for this value of dust opacity was motivated by the analysis of the attenuation-inclination relation by Driver et al. (2007), who found an average dust opacity for local universe disk galaxies of $\tau_{B}^{f}=3.8$. A similar average value for comparable stellar masses was also found by Grootes et al. (2013a). Moreover, radiative transfer analysis of the UV to FIR SEDs of individual edge-on galaxies by Misiriotis et al. (2001) and Popescu et al. (2004) found similar values for $\tau_{B}^{f}$.

In the left panel of Fig. 25 we show the comparison of our model predictions with the data. Overall, the data show the same monotonic increase in disk sizes with inclination as predicted by our model. The main contributor to the inclination dependence is due to the effect of dust on the single disk. This can be seen by looking at similar plots, where we omitted the corrections for projection effects corr ${ }^{\text {proj }}$ (middle panel in Fig. 25), and the decomposition effects $\operatorname{corr}^{B / D}$ (right panel in Fig. 25). In each of the latter cases, the agreement with the data is slightly worse. In particular the projection and decomposition effects account for the steepening of the inclination dependence at higher inclinations. However, the latter effects are within the observational errors of the data.

Using the same sample of 33770 galaxies considered for the above comparison, we used the same approach to study the inclination dependence of bulge effective radii. We derived the average bulge effective radius for each bin in inclination (this time considering larger bins with a size of 0.2 to reduce the noise in the data), while for the model predictions the whole chain of corrections was again considered:

$\operatorname{corr}\left(R_{\mathrm{b}}^{\mathrm{eff}}\right)=\operatorname{corr}^{\mathrm{proj}}\left(R_{\mathrm{b}}^{\mathrm{eff}}\right) * \operatorname{corr}^{\text {dust }}\left(R_{\mathrm{b}}^{\mathrm{eff}}\right) * \operatorname{corr}^{B / D}\left(R_{\mathrm{b}}^{\mathrm{eff}}\right)$

where $R_{\mathrm{b}}^{\text {eff }}$ is the effective radius of the stellar bulge. As before (disks), the model predictions are made for $\tau_{B}^{f}=4$. In Fig. 26, we present the result of this comparison. Unlike the case of the disks, the data are noisier. This may possibly be due to the limited angular resolution of the SDSS data 

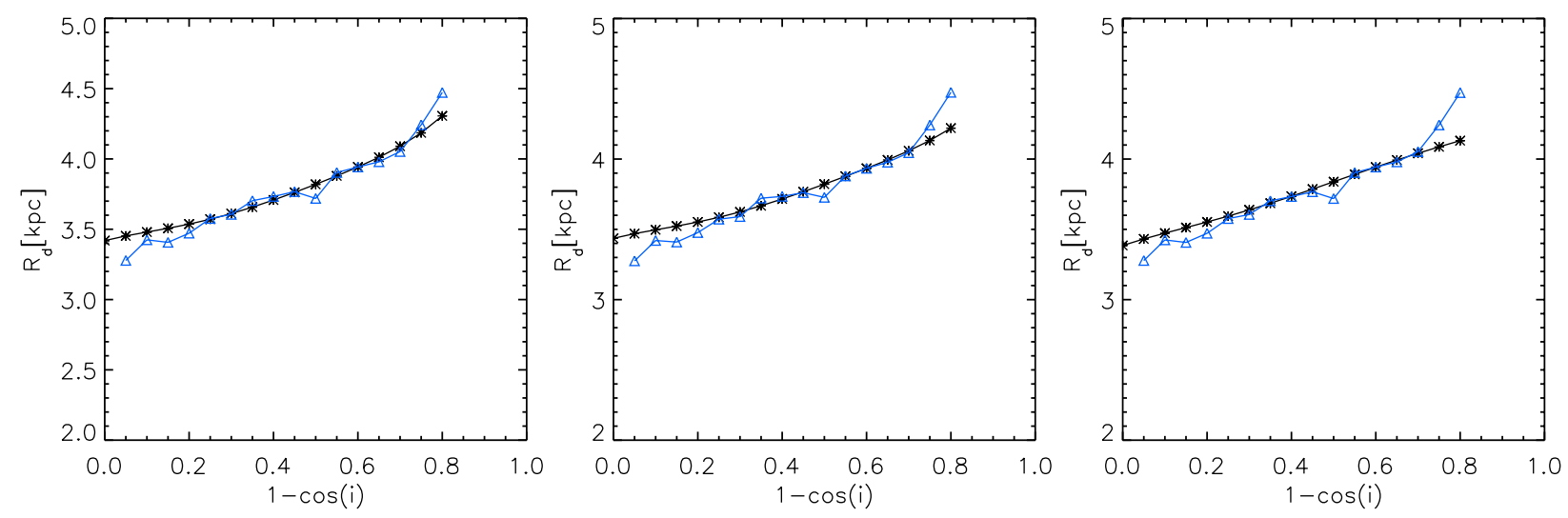

Fig. 25. Left: average inclination dependence of disk sizes for a sample of galaxies selected from Simard et al. (2011) (blue curve). Overplotted in black are the predictions of our model for a disk population, scaled to the averaged disk size derived from the data, at $1-\cos (i)=0.6$, which corresponds to an intrinsic value of $3.05 \mathrm{kpc}$. Middle: the same, but with the projection effects corr ${ }^{\text {proj }}$ not included in the chain of corrections. Right: the same, but with decomposition effects $\operatorname{corr}^{B / D}$ not included in the chain of corrections.

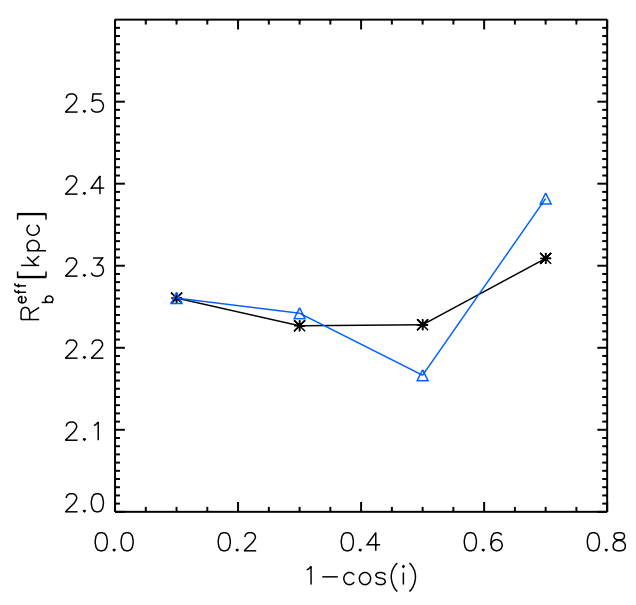

Fig. 26. Average inclination dependence of bulge effective radii for a sample of galaxies selected from Simard et al. (2011) (blue curve). Overplotted in black are the predictions of our model for a bulge population, scaled to the averaged bulge effective radius derived from the data, at $1-\cos (i)=[0.0,0.2]$.

of 1.4" FWHM (Abazajian et al. 2009), which corresponds to $2.3 \mathrm{kpc}$ at the 0.08 redshift limit of the sample considered in this paper. Nonetheless, both the data and the model show a roughly flat dependence with inclination. It is interesting to note that our model predictions for $\tau_{B}^{f}=4$ (considered to be representative for local universe galaxies) can account for the contrasting inclination dependence of scale-length of disks and effective radius of bulges.

To conclude, while on average our model can account for the trends seen in the data, a more detailed analysis of the inclination dependence of disk and bulge sizes would require both a more accurate determination of disk scale-lengths and bulge effective radii and an analysis done on an object-by-object case. From the point of view of the data, a more accurate determination of sizes would require higher resolution images, as will soon become available from VISTA/VST. From the point of view of the analysis, corrections to each data point should be applied, according to the dust opacity of each galaxy. This, in turn, requires determination of $\tau_{B}^{f}$. For galaxies with available panchromatic integrated luminosity densities, determination of $\tau_{B}^{f}$ can be obtained by using the library of radiative transfer model SEDs of Popescu et al. (2011), the same model that was used to derive the dust corrections in this paper and in Pastrav et al. (2013). Since the fits to the SEDs need to be scaled according to the size of the disk, this becomes an iterative problem to solve. The use of this approach allows for a self-consistent determination of both intrinsic parameters of galaxies derived from global measurements and structural parameters derived from images. For galaxies without measurements of integrated dust luminosities, the dust opacity can be derived solely from optical data, using the method of Grootes et al. (2013a), which was calibrated by using the same radiative transfer model of Popescu et al. (2011), again allowing for a self-consistent analysis of both integrated quantities and structural properties.

\section{Summary}

In this paper we have presented the results of a study to quantify the effects of dust and projection effects on the derived photometric parameters of disks and bulges obtained from bulge-disk decomposition. As discussed in Pastrav et al. (2013), these effects can be separated from the effects of dust and projection effects on single disks and bulges. Thus, in this paper we have only analysed the above mentioned effects on the decomposition itself.

We used simulated images calculated with radiative transfer techniques. The simulations were produced as part of the large library of dust and PAH emission SEDs and corresponding dust attenuations presented in Popescu et al. (2011). All the simulations were calculated using a modified version of the ray-tracing radiative transfer code of Kylafis \& Bahcall (1987).

We fitted the simulated images with 1D analytic functions available in GALFIT. The following types of fits were considered: i) fits combining an infinitely thin exponential plus a variable-index Sérsic function for the disk and bulge component, respectively, and ii) fits combining variable-index Sérsic functions for both the disk and the bulge.

The main effects dust has on the bulge-disk decomposition are as follows:

\section{Galaxies with exponential bulges}

- The derived scale-length of a decomposed disk (obtained from fits of type i.) is smaller than the derived scale-length of a single disk (in the absence of a bulge).

- The derived axis-ratio of a decomposed disk (obtained from fits of type i.) is not changed in the decomposition process. 
- The derived effective radius of a decomposed bulge (obtained from fits of type i.) is smaller than the effective radius of a single bulge (in the absence of a disk).

- The derived Sérsic index of a decomposed bulge (obtained from fits of type i.) is slightly smaller than that obtained in the absence of a disk.

- The derived bulge-to-disk ratio (obtained from fits of type i.) is smaller that that obtained from single components.

- The derived effective radius of a decomposed disk (obtained from fits of type ii.) is closer to the single disk solution (in the absence of a bulge).

- The derived axis-ratio of a decomposed disk (obtained from fits of type ii.) is not changed in the decomposition process.

- The derived effective radius of a decomposed bulge (obtained from fits of type ii.) is close to the effective radius of a single bulge (in the absence of a disk).

- The derived bulge-to-disk ratio (obtained from fits of type ii.) is relatively unchanged in the decomposition process.

- The corrections $\operatorname{corr}^{\text {dust } B / D}$ are relatively insensitive to the exact value of the $B / D$.

\section{Galaxies with de Vaucouleurs bulges}

- The overall trends are the same as those for exponential bulges.

- The amplitude of the corrections $\operatorname{corr}^{\text {dust }, B / D}$ is larger than for the case of systems with exponential bulges.

The predictions for the inclination dependence of disk scalelengths and bulge effective radii were compared with observational data from a sample selected from Simard et al. (2011). We show that on average our model can account for the trends seen in the data. We also show that the main contributor to the steep increase of disk scale-length with inclination is the effect of dust on single disks, while projection and decomposition effects produce only secondary effects, strengthening the agreement with the data. We recommend that for more detailed studies of sizes, an analysis on an object-by-object case should be involved, in conjunction with determinations of disk opacities. We show that self-consistent determinations of both intrinsic disk and bulge sizes and dust opacities can be obtained using the library of model SEDs of Popescu et al. (2011) or the method of Grootes et al. (2013a), since these have been obtained with the same radiative transfer model that was used to derived the corrections presented in this paper.

Acknowledgements. We would like to acknowledge the referee for his/her very useful comments and suggestions.

\section{References}

Abazajian, K. N., Adelman-McCarthy, J. K., Ageros, M. A., et al. 2009, ApJS, 182,543

Allen, P. D., Driver, S. P., Graham, A. W., et al. 2006, MNRAS, 371, 2

Benson, A., Džanović, D., Frenk, C. S., \& Sharples, R. 2007, MNRAS, 379, 841
Bernardi, M., Meert, A., Vikram, V., et al. 2012, MNRAS, submitted [arXiv: 1211.6122]

Bourne, N., Maddox, S. J., Dunne, L., et al. 2012, MNRAS, 421, 3027

Bruce, V. A., Dunlop, J. S., Cirasuolo, M., et al. 2012, MNRAS, 427, 1666

Cameron, E., Driver, S., Grahah, A. W., \& Liske, J. 2009, ApJ, 699, 105

Casaponsa, B., Heavens, A. F., Kitching, et al. 2013, MNRAS, 430, 2844

Ciotti, L., \& Bertin, G. 1999, A\&A, 352, 447

de Jong, J. T. A., Verdoes Kleijn, G. A., Kuijken, K. H., \& Valentijn, E. A. 2012, ExA, 35, 25

Dale, D. A., Aniano, G., Engelbracht, C. W., et al. 2012, ApJ, 745, 95

Dariush, A., Cortese, L., Eales, S., et al. 2011, MNRAS, 418, 64

Draine, B. T., \& Li, A. 2007, ApJ, 657, 810

Driver, C. C., Popescu, C. C., Tuffs, R. J., et al. 2007, MNRAS, 379, 1022

Driver, S. P., Hill, D. K., Kelvin, L. S., et al. 2011, MNRAS, 413, 971

Fabricius, M. H., Saglia, R. P., Fisher, D. B., et al. 2012, ApJ, 754, 67

Fisher, D. B., \& Drory, N. 2008, ApJ, 136, 773

Gadotti A. D. 2008, MNRAS, 384, 420

Gadotti, A. D. 2009, MNRAS, 393, 1531

Gadotti, A. D., Baes, M., \& Falony, S. 2010, MNRAS, 403, 2053

Graham, A. W. 2001, AJ, 121, 820

Graham, A. W., \& Driver, S. P. 2005, PASA, 22, 118

Graham, A. W., \& Driver, S. P. 2007a, ApJ, 655, 77

Graham, A. W., \& Driver, S. P. 2007b, MNRAS, 380, L15

Graham, A. W., Driver, S. P., Allen, P. D., \& Liske, J. 2007, MNRAS, 378, 198

Grootes, M., Tuffs, R. J., Popescu, C. C., et al. 2013a, ApJ, 766, 59

Grootes, M. W., Tuffs, R. J., Popescu, C. C., et al. 2013b, MNRAS, in press

Häußler, B., Bamford, S. P., Vika, M., et al. 2013, MNRAS, 430, 330

Hoyos, C., den Brok, M., Verdoes, K. G., et al. 2011, MNRAS, 411, 2439

Kelvin, L. S., Driver, S. P., Robotham, A. S. G., et al. 2012, MNRAS, 421, 1007

Kylafis, N. D., \& Bahcall, J. N. 1987, ApJ, 317, 637

Lackner, C. N., \& Gunn, J. E. 2012, MNRAS, 421, 2277

Laureijs, R. J., Duvet, L., Escudero, S. I., et al. 2010, SPIE, 7731, 40

Maltby, D. T., Hoyos, C., Gray, M. E., Aragón-Salamanca, A., \& Wolf, C. 2012, MNRAS, 420, 2475

Misiriotis, A., Popescu, C. C., Tuffs, R., \& Kylafis, N. D. 2001, A\&A, 372, 775

Möllenhoff, C. 2004, A\&A, 415, 63

Möllenhoff, C., \& Heidt, J. 2001, A\&A, 368, 16

Möllenhoff, C., Appenzeller, I., Gässler, W., et al. 1999, A\&A, 352, L5

Möllenhoff, C., Popescu, C. C., \& Tuffs, R. J. 2006, A\&A, 456, 941

Pastrav, B. A., Popescu, C. C., Tuffs, R. J., \& Sansom, A. E. 2012, in The Spectral Energy Distribution of Galaxies, eds. R. J. Tuffs, \& C. C. Popescu, Proc. IAU Symp., 284, 306

Pastrav, B. A., Popescu, C. C., Tuffs, R. J., \& Sansom, A. E. 2013, A\&A, 553, A80

Peng, C. Y., Ho, L. C., Impey, C. D., \& Rix, H.-W. 2002, AJ, 124, 266

Peng, C. Y., Ho, L. C., Impey, C. D., \& Rix, H.-W. 2010, AJ, 139, 2097

Popescu, C. C., Misiriotis, A., Kylafis, N. D., Tuffs, R. J., \& Fischera, J. 2000, A\&A, 362, 138

Popescu, C. C., Tuffs, R. J., Völk, H. J., Pierini, D., \& Madore, B. F. 2002, ApJ, 567,221

Popescu, C. C., Tuffs, R. J., Kylafis, N. D., \& Madore, B. F. 2004, A\&A, 414, 45

Popescu, C. C., Tuffs, R. J., Dopita, M. A., et al. 2011, A\&A, 527, A109

Rowlands, K., Dunne, L., Maddox, S., et al. 2012, MNRAS, 419, 2545

Savorgnan, G., Graham, A. W., Marconi, A., et al. 2013, MNRAS, in press [arXiv: 1306.2679]

Simard, L., Willmer, C. N. A., Vogt, N. P., et al. 2002, ApJS, 142, 1

Simard, L., Mendel, J.T, Patton, D. R., et al. 2011, ApJS, 196, 11

The Dark Energy Survey Collaboration, Abbott, T., Alderning, G., Annis, J., et al. 2005 [arXiv: astro-ph/0510346]

Tuffs, R. J., Popescu, C. C., Pierini, D., et al. 2002, ApJS, 139, 37

Tuffs, R. J., Popescu, C. C., Völk, H. J., Kylafis, N. D., \& Dopita, M. A. 2004, A\&A, 419, 821

Vika, M., Bamford, S. P., Häßler, B., et al. 2013, MNRAS, in press [arXiv: 1307.4996]

Vlahakis, C., Dunne, L., \& Eales, S. 2005, MNRAS, 364, 1253

Weingartner, J. C., \& Draine, B. T. 2001, ApJ, 548, 296

York, D., Adelmam, J., Anderson, J. E., et al. 2000, AJ, 120, 1579 


\section{Appendix A: Examples of projection effects on decomposed disks and bulges for galaxies with exponential bulges and $B / D=0.5$}
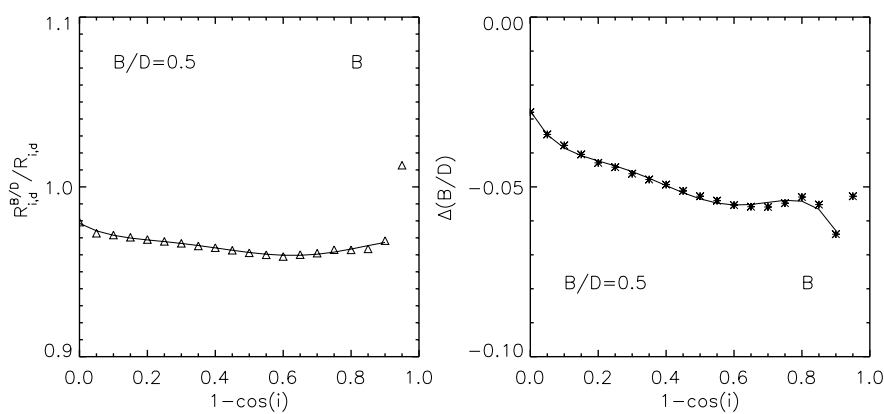

Fig. A.1. Left: projection effects on the derived scale-length of decomposed disks for $B / D=0.50$. The symbols represent the measurements while the solid lines are polynomial fits to the measurements. The plots represent the ratio between the intrinsic scale-lengths of decomposed and single disks, $R_{i, \mathrm{~d}}^{B / D}$ and $R_{i, \mathrm{~d}}$, respectively, as a function of inclination $(1-\cos (i))$, for the $B$-band. An exponential (disk) plus a variable index Sérsic (bulge) distribution were used for image decomposition. Right: as in the left panel, but for the derived bulge-to-disk ratios, $B / D$. The effects are represented as differences between the intrinsic $B / D$ of decomposed disks and bulges and those of single disks and bulges.
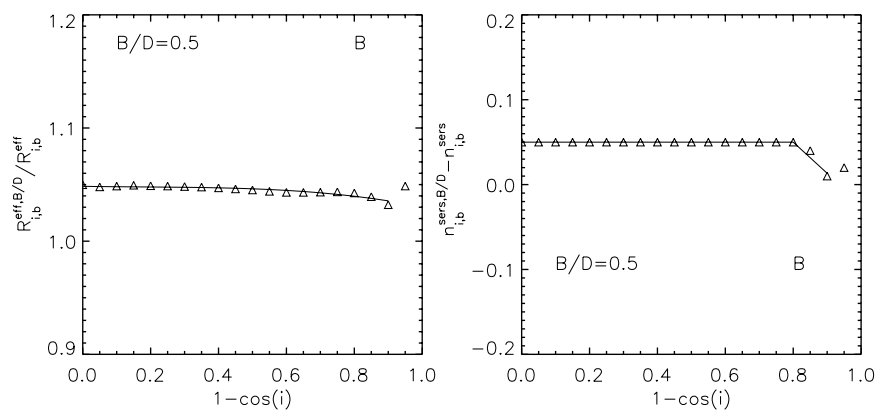

Fig. A.2. As in Fig. A.1, but for the derived effective radius $R_{i, \mathrm{~b}}^{\text {eff, } B / D}$ (left) and for the derived Sérsic indices (right) of decomposed exponential bulges. The effects on Sérsic indices are represented as differences between the measured Sérsic index of decomposed and single bulges, $n_{i, \mathrm{~b}}^{\text {sers, } B / D}$ and $n_{i, \mathrm{~b}}^{\text {sers }}$, respectively.
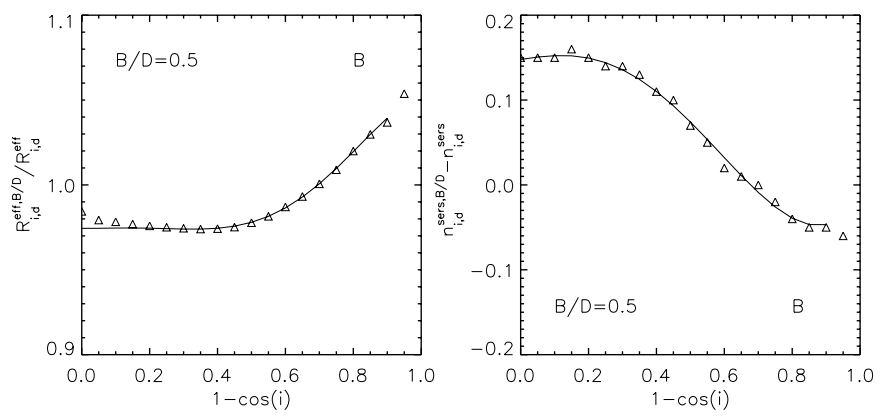

Fig. A.3. Left: projection effects on the derived effective radius of decomposed disks for $B / D=0.5$. The symbols represent the measurements while the solid lines are polynomial fits to the measurements. The plots represent the ratio between the intrinsic effective radius of decomposed and single disks, $R_{i, \mathrm{~d}}^{\text {eff, } B / D}$ and $R_{i, \mathrm{~d}}^{\text {eff }}$, respectively, as a function of inclination $(1-\cos (i))$, for the $B$-band. Two variable index Sérsic functions were used for image decomposition. Right: as in the left panel, but for the derived Sérsic index, $n^{\text {sers }}$. The effects are represented as differences between the measured Sérsic index of decomposed and single disks, $n_{i, \mathrm{~d}}^{\text {sers, } B / D}$ and $n_{i, \mathrm{~d}}^{\text {sers }}$, respectively.
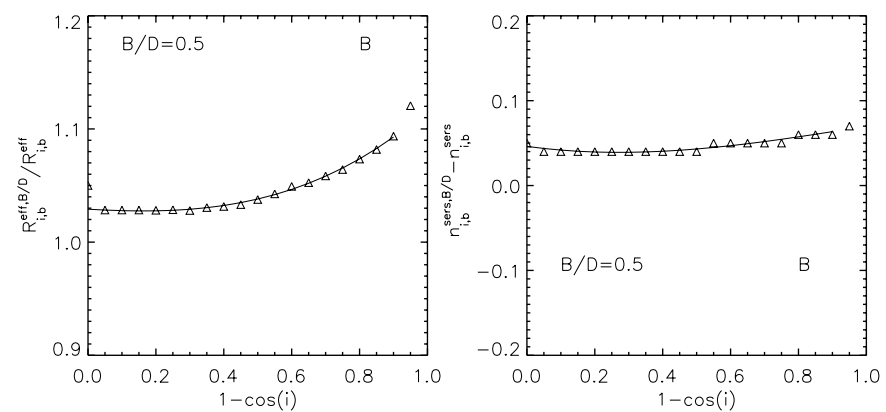

Fig. A.4. As in Fig. A.3, but for the decomposed exponential bulges.

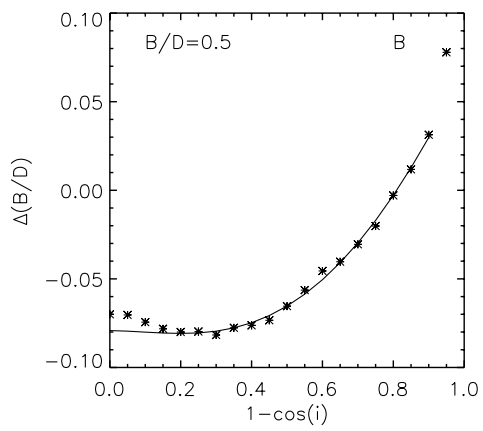

Fig. A.5. As in Fig. A.1, right, but for fits done with two variable Sérsic index functions.

\section{Appendix B: Examples of projection effects on decomposed disks and bulges for galaxies with de Vaucouleurs bulges and $B / D=0.25$}
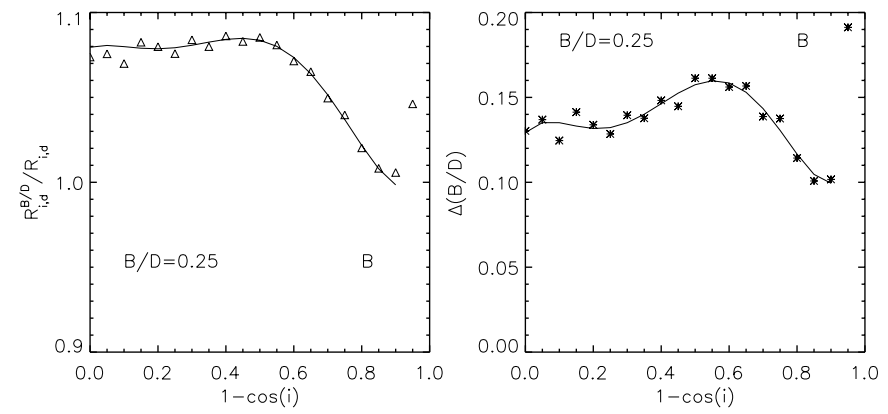

Fig. B.1. Left: projection effects on the derived scale-length of decomposed disks for $B / D=0.25$. The symbols represent the measurements while the solid lines are polynomial fits to the measurements. The plots represent the ratio between the intrinsic scale-lengths of decomposed and single disks, $R_{i, \mathrm{~d}}^{B / D}$ and $R_{i, \mathrm{~d}}$, respectively, as a function of inclination $(1-\cos (i))$, for the $B$-band. An exponential (disk) plus a variable index Sérsic (bulge) distribution were used for image decomposition. Right: as in the left panel, but for the derived bulge-to-disk ratios, $B / D$. The effects are represented as differences between the intrinsic $B / D$ of decomposed disks and bulges and those of single disks and bulges. 
B. A. Pastrav et al.: The effects of dust on the photometric parameters of decomposed disks and bulges
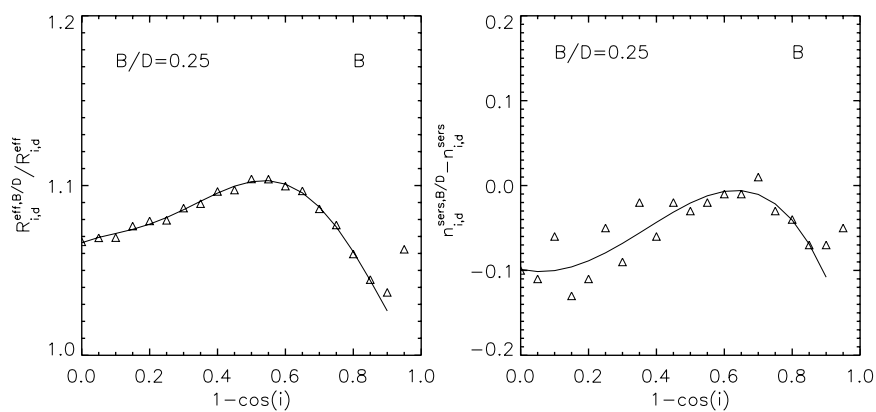

Fig. B.2. Left: projection effects on the derived effective radius of decomposed disks for $B / D=0.25$. The symbols represent the measurements while the solid lines are polynomial fits to the measurements. The plots represent the ratio between the intrinsic effective radius of decomposed and single disks, $R_{i, \mathrm{~d}}^{\text {eff } B / D}$ and $R_{i, \mathrm{~d}}^{\mathrm{eff}}$, respectively, as a function of inclination $(1-\cos (i))$, for the $B$-band. Two variable index Sérsic functions were used for image decomposition. Right: as in the left panel, but for the derived Sérsic index, $n^{\text {sers }}$. The effects are represented as differences between the measured Sérsic index of decomposed and single disks, $n_{i, \mathrm{~d}}^{\text {sers, } B / D}$ and $n_{i, \mathrm{~d}}^{\text {sers }}$, respectively.

\section{Appendix C: Example of dust effects \\ on decomposed disks and bulges for galaxies with exponential bulges and $B / D=0.5$}

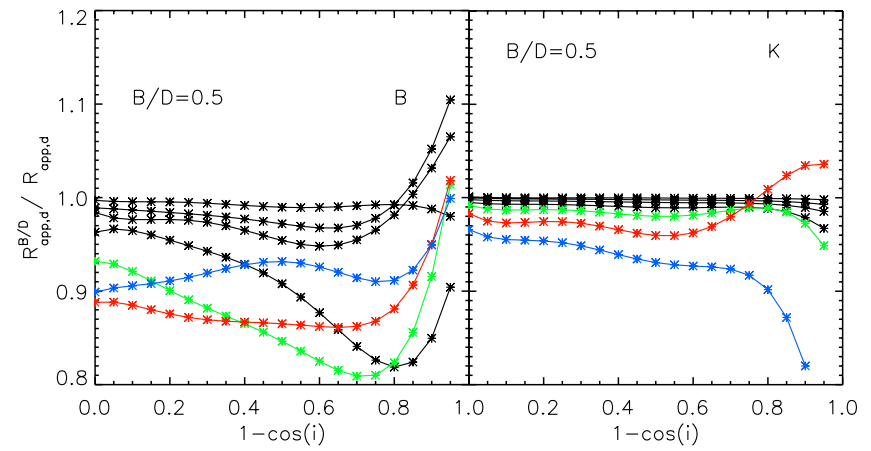

Fig. C.1. Dust effects $\left(\operatorname{corr}^{B / D}-\operatorname{corr}^{\mathrm{proj}, B / D}\right)$ on the derived scale-length of decomposed disks for $B / D=0.5$. The solid lines are polynomial fits to the measurements. The plots represent the ratio between the apparent scale-lengths of decomposed and single disks, $R_{\text {app,d }}^{B / D}$ and $R_{\text {app,d }}$, respectively, as a function of inclination $(1-\cos (i))$, for the $B$ and $K$ optical bands. An exponential (disk) plus a variable index Sérsic (bulge) distribution were used for image decomposition. The black curves are plotted for $\tau_{B}^{f}=0.1,0.3,0.5,1.0$, while the other curves correspond to $\tau_{B}^{f}=2.0$ (green), 4.0 (red) and 8.0 (blue)

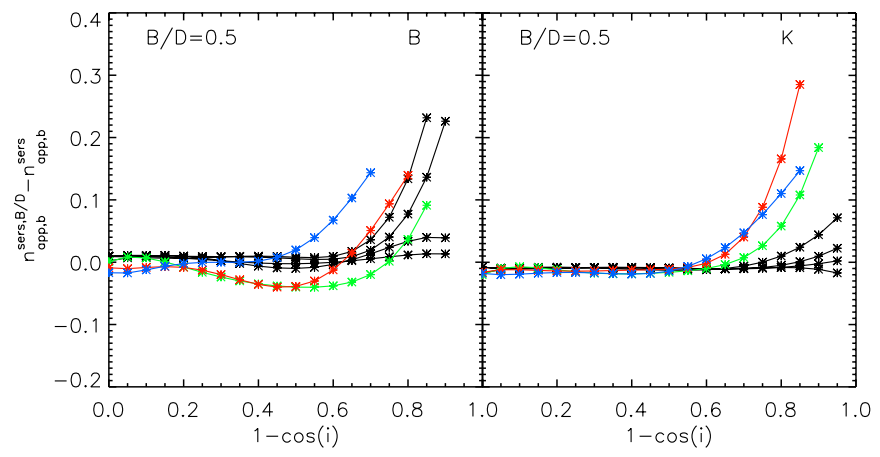

Fig. C.2. As in Fig. C.1, but for the derived Sérsic index of decomposed exponential bulges. The effects are represented as differences between the measured Sérsic index of decomposed and single bulges, $n_{\mathrm{app}, \mathrm{b}}^{\text {ser, } B D}$ and $n_{\text {app, b }}^{\text {sers }}$, respectively.

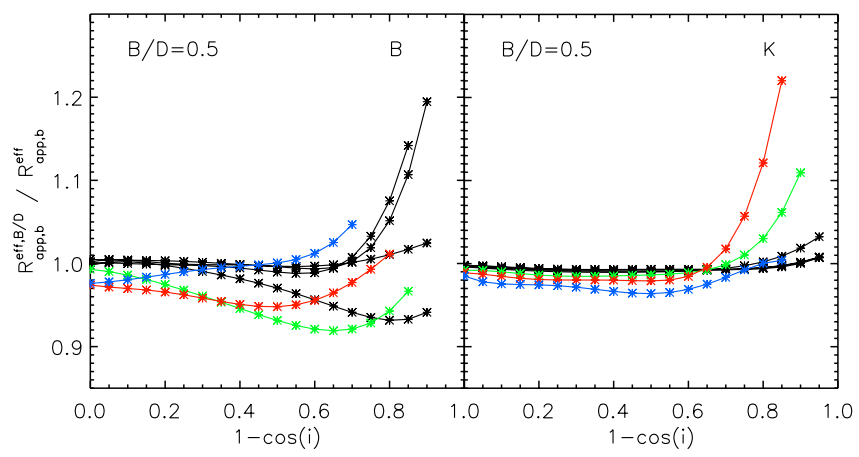

Fig. C.3. As in Fig. C.1, but for the derived effective radius of decomposed exponential bulges.

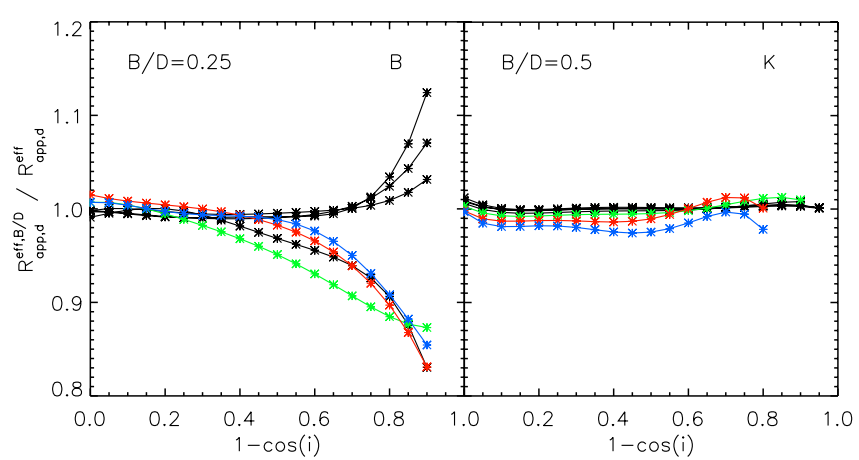

Fig. C.4. Dust effects $\left(\operatorname{corr}^{B / D}-\operatorname{corr}^{\mathrm{proj}, B / D}\right)$ on the derived efective radius of decomposed disks, for $B / D=0.5$. The solid lines are polynomial fits to the measurements. The plots represent the ratio between the apparent effective radius of decomposed and single disks, $R_{\mathrm{app}, \mathrm{d}}^{\mathrm{eff}, B}$ and $R_{\mathrm{app}, \mathrm{d}}^{\mathrm{eff}}$, respectively, as a function of inclination $(1-\cos (i))$, for the $B$ and $K$ optical bands. Two variable Sérsic index functions were used for image decomposition. The black curves are plotted for $\tau_{B}^{f}=0.1,0.3,0.5,1.0$, while the other curves correspond to $\tau_{B}^{f}=2.0$ (green), 4.0 (red) and 8.0 (blue).

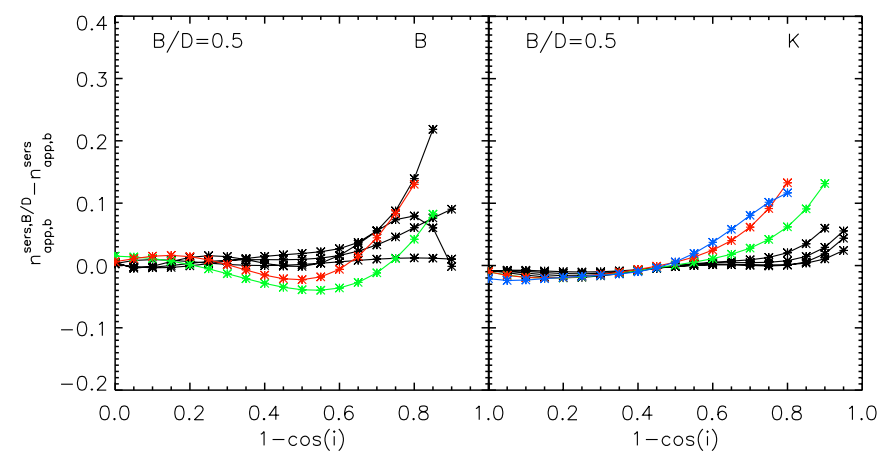

Fig. C.5. As in Fig. C.4, but for the derived Sérsic index of decomposed exponential bulges. The effects are represented as differences between the measured Sérsic index of decomposed and single bulges, $n_{\mathrm{app}, \mathrm{b}}^{\mathrm{ser}, B / D}$ and $n_{\text {app, }}^{\text {sers }}$, respectively. 


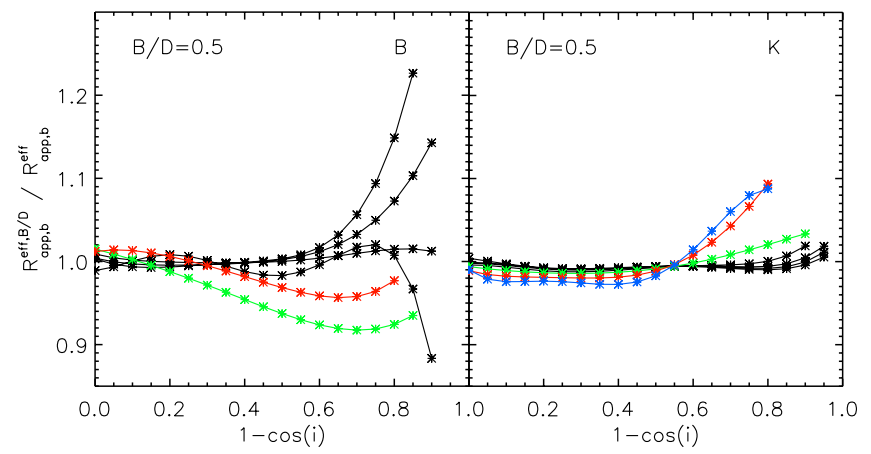

Fig. C.6. As in Fig. C.4, but for the derived effective radius of decomposed exponential bulges.

\section{Appendix D: Examples of dust effects \\ on decomposed disks and bulges for galaxies with de Vaucouleurs bulges and $B / D=0.25$}

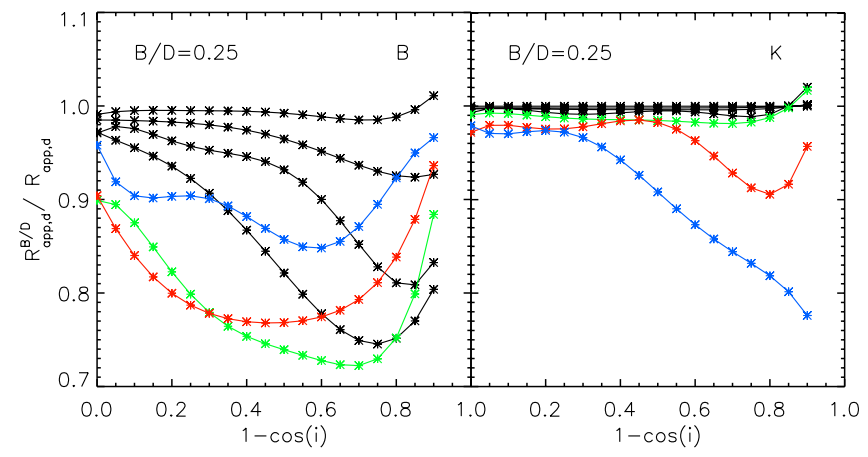

Fig. D.1. Dust effects $\left(\operatorname{corr}^{B / D}-\operatorname{corr}^{\mathrm{proj}, B / D}\right)$ on the derived scale-length of decomposed disks for $B / D=0.25$. The solid lines are polynomial fits to the measurements. The plots represent the ratio between the apparent scale-lengths of decomposed and single disks, $R_{\text {app,d }}^{B / D}$ and $R_{\text {app,d }}$, respectively, as a function of inclination $(1-\cos (i))$, for the $B$ and $K$ optical bands. An exponential (disk) plus a variable index Sérsic (bulge) distribution were used for image decomposition. The curves are plotted for $\tau_{B}^{f}=0.1,0.3,0.5,1.0$ (black), 2.0 (green), 4.0 (red) and 8.0 (blue).

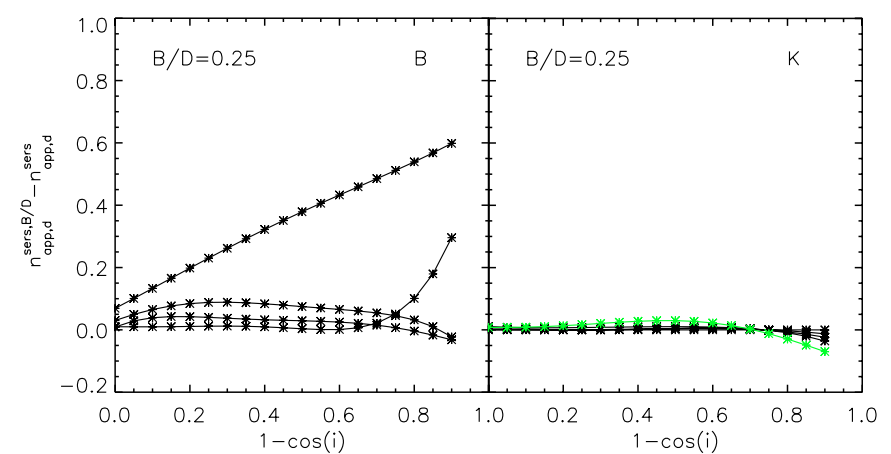

Fig. D.2. Dust effects $\operatorname{corr}^{B / D}$ on the derived Sérsic index of decomposed disks, for $B / D=0.25$. The solid lines are polynomial fits to the measurements. The plots represent the difference between the derived Sérsic index of decomposed and single disks, $n_{\text {app,d }}^{\text {sers } B / D}$ and $n_{\text {app,d }}^{\text {sers }}$, respectively, as a function of inclination $(1-\cos (i))$, for the $B$ and $K$ optical bands. Two variable Sérsic index functions were used for image decomposition. The curves are plotted for $\tau_{B}^{f}=0.1,0.3,0.5,1.0$ (black), 2.0 (green) and 4.0 (red).

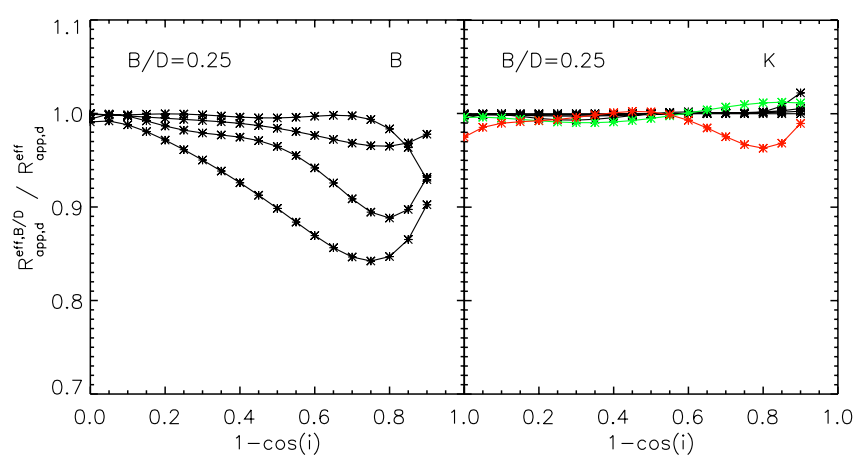

Fig. D.3. As in Fig. D.2, but for derived effective radii of decomposed disks. 